\title{
Two types of condition for the global stability of delayed SIS epidemic models with nonlinear birth rate and disease induced death rate
}

\author{
Yukihiko Nakata ${ }^{\text {a,b }}$ Yoichi Enatsu ${ }^{\text {a }}$ \\ ${ }^{a}$ Department of Pure and Applied Mathematics, Waseda University, 3-4-1 Okubo, Shinjuku-ku, Tokyo, 169-8555, Japan \\ ${ }^{\mathrm{b}}$ BCAM - Basque Center for Applied Mathematics Bizkaia Technology Park, Building 500, E-48160, Derio, Basque \\ Country, Spain \\ Yoshiaki Muroya ${ }^{\mathrm{c}} 1$ \\ ${ }^{\mathrm{c}}$ Department of Mathematics, Waseda University 3-4-1 Ohkubo, Shinjuku-ku, Tokyo 169-8555, Japan
}

\begin{abstract}
We study global asymptotic stability for an SIS epidemic model with maturation delay proposed by Cooke et al. [1]. It is assumed that the population has a nonlinear birth term and disease causes death of infective individuals. By using a monotone iterative method, we establish sufficient conditions for the global stability of an endemic equilibrium when it exists dependently on the monotone property of the birth rate function. Based on the analysis, we, further, study the model with two specific birth rate functions $B_{1}(N)=b \mathrm{e}^{-a N}$ and $B_{3}(N)=A / N+c$, where $N$ denotes the total population. For each model, the disease induced death rate which guarantees the global stability of the endemic equilibrium is established and this gives a positive answer for an open problem by Zhao and Zou [5].
\end{abstract}

Key words: SIS epidemic models; nonlinear birth rate function; disease induced death rate; global asymptotic stability; the basic reproduction number; permanence

\section{Introduction}

Cooke et al. [1] derived a population growth model for single-species with multiple life stages as follows.

$$
N^{\prime}(t)=B(N(t-T)) N(t-T) \mathrm{e}^{-d_{1} T}-d N(t)
$$

\footnotetext{
${ }^{1}$ Research is partially supported by Scientific Research (c), No.21540230 of Japan Society for the Promotion of Science. Email addresses: yunayuna.na@gmail.com, nakata@bcamath.org (Yukihiko Nakata), yo1.gc-rw.docomo@akane.waseda.jp (Yoichi Enatsu), ymuroya@waseda.jp (Yoshiaki Muroya).
} 
where $^{\prime}=\frac{d}{d t}, N(t)$ is the adult (matured) population size at time $t, d>0$ is the death rate at the adult stage, $B(N)$ is a birth rate function, $T$ is the developmental or maturation time and $d_{1}$ is the death rate for each life stage prior to the adult stage. Typical examples of the birth rate functions are

$$
\begin{aligned}
& \text { (B1) } B_{1}(N)=b \mathrm{e}^{-a N}, a>0, \\
& \text { (B2) } B_{2}(N)=\frac{p}{q+N^{n}}, p>0, q>0, n>0, \\
& \text { (B3) } B_{3}(N)=\frac{A}{N}+c, A>0, c>0 .
\end{aligned}
$$

Functions $B_{1}(N)$ and $B_{2}(N)$ with $n=1$ are known as the Ricker type and the Beverton Holt type, respectively. $B_{3}(N) N$ denotes a constant immigration rate with a linear birth term $c N$. Cooke et al. [1] investigated the dynamics of (1.1). They established global asymptotic stability of a unique positive equilibrium if it exists by assuming that the birth rate function satisfies suitable monotone properties. The maturation delay changes dynamics and periodic solution was observed when the birth rate function is the Ricker type. Population model, which has taken the maturation delay into the consideration, has been studied by many authors (see [2-4] and the references therein)

Moreover, Cooke et al. [1] introduced disease into (1.1) and divided the population into two classes, susceptible and infective individuals. Then, the following SIS epidemic model is obtained.

$$
\left\{\begin{array}{l}
S^{\prime}(t)=B(N(t-T)) N(t-T) \mathrm{e}^{-d_{1} T}-d S(t)-\frac{\lambda S(t) I(t)}{N(t)}+\gamma I(t), \\
I^{\prime}(t)=\frac{\lambda S(t) I(t)}{N(t)}-(d+\varepsilon+\gamma) I(t),
\end{array}\right.
$$

where $S(t)$ is the susceptible population, $I(t)$ is the infective population and $N(t)=S(t)+I(t)$ is the total population at time $t \geq T$. $\varepsilon \geq 0$ is the disease induced death rate, $\gamma \geq 0$ is the recovery rate and $\lambda>0$ is the contact rate. From (1.2), we also obtain the following equation.

$$
\left\{\begin{array}{l}
I^{\prime}(t)=\lambda(N(t)-I(t)) \frac{I(t)}{N(t)}-(d+\varepsilon+\gamma) I(t), \\
N^{\prime}(t)=B(N(t-T)) N(t-T) \mathrm{e}^{-d_{1} T}-d N(t)-\varepsilon I(t) .
\end{array}\right.
$$

If we assume

- (H1) $B(N) \in C^{1}((0,+\infty),(0,+\infty))$ with $B^{\prime}(N)<0$ for all $N \in(0,+\infty), B(0+)>(d+\varepsilon) \mathrm{e}^{d_{1} T} \geq$ $d \mathrm{e}^{d_{1} T}>B(+\infty)$ and there exists a $G(N) \in C^{1}((0,+\infty),(0,+\infty))$ such that $G(N)=B(N) N$ for all $N>0$,

then (1.3) has two possible equilibria, a disease free equilibrium $E_{0}=\left(0, B^{-1}\left(d \mathrm{e}^{d_{1} T}\right)\right)$ and an endemic equilibrium $E_{\varepsilon}^{*}=\left(I^{*}(\varepsilon), N^{*}(\varepsilon)\right)$, where

$$
I^{*}(\varepsilon)=\left(1-\frac{1}{R_{0}(\varepsilon)}\right) N^{*}(\varepsilon), N^{*}(\varepsilon)=B^{-1}\left(\left(d+\varepsilon\left(1-\frac{1}{R_{0}(\varepsilon)}\right)\right) \mathrm{e}^{d_{1} T}\right) .
$$

More precisely, if $R_{0}(\varepsilon) \leq 1$, then the disease free equilibrium $E_{0}$ is the only equilibrium and if $R_{0}(\varepsilon)>1$, then the disease free equilibrium $E_{0}$ and the endemic equilibrium $E_{\varepsilon}^{*}$ exist for (1.3) where

$$
R_{0}(\varepsilon)=\frac{\lambda}{d+\varepsilon+\gamma}
$$


$R_{0}(\varepsilon)$ is called the basic reproduction number for (1.3) which gives the average number of new infectives produced by one infective during the mean death adjusted infective period (see also [1, Theorem 4.2]).

To investigate (1.1), we set a suitable phase space. We denote by $C=C\left([-T, 0], \mathbb{R}^{2}\right)$ the Banach space of continuous functions mapping the interval $[-T, 0]$ into $\mathbb{R}^{2}$ equipped with the sup-norm. The nonnegative cone of $C$ is defined as $C_{+}=C\left([-T, 0], \mathbb{R}_{+}^{2}\right)$. From the biological meanings, the initial condition for (1.1) is $I(\theta)=\phi_{1}(\theta), N(\theta)=\phi_{2}(\theta)$ for $\theta \in[-T, 0]$ where $\left(\phi_{1}, \phi_{2}\right) \in C_{+}$. We set

$$
\left\{\begin{array}{l}
X=\left\{\left(\phi_{1}, \phi_{2}\right) \in C_{+}: \phi_{2}(\theta) \geq \phi_{1}(\theta), \text { for all } \theta \in[-T, 0]\right\}, \\
X_{0}=\left\{\left(\phi_{1}, \phi_{2}\right) \in X: \phi_{1}(0)>0\right\},
\end{array}\right.
$$

and assume that the initial conditions satisfy $(I(\theta, \phi), N(\theta, \phi))=\phi(\theta)$ for all $\theta \in[-T, 0]$ and $\phi=\left(\phi_{1}, \phi_{2}\right) \in X_{0}$.

Further, if we assume

- (H2) $G^{\prime}(N)=\frac{d}{d N}(B(N) N)>0$, for all $N \in(0,+\infty)$, or $G(N)=B(N) N$ is bounded on $(0,+\infty)$ and positive equilibrium $N^{*}(0)=B^{-1}\left(d \mathrm{e}^{d_{1} T}\right)$ of (1.1), is globally asymptotically stable for initial values in $C\left([-T, 0], \mathbb{R}^{+}\right) \backslash\{0\}$,

then, the disease free equilibrium $E_{0}$ of (1.1) is globally asymptotically stable in the absence of disease (see [1, Theorems 3.1,3.3]). Thus, the population is stable at the disease free equilibrium $E_{0}$, if there is no disease in the host population.

Cooke et al. [1] showed that $R_{0}(\varepsilon)$ works as a global threshold parameter for the cases i) $T=0$ and $\varepsilon \geq 0$ (there is no maturation delay and disease may induce the death of the infective) and ii) $T>0$ and $\varepsilon=0$ (there is maturation delay and disease does not induce the death of the infective) under the assumptions (H1) and (H2) (see [1, Theorems 4.1, 4.3 and 4.4]). More precisely, if $R_{0}(\varepsilon)<1$, then the disease free equilibrium $E_{0}$ is globally asymptotically stable and if $R_{0}(\varepsilon)>$ 1 , then the endemic equilibrium $E_{\varepsilon}^{*}$ exists and is globally asymptotically stable in these cases. Furthermore, they showed the local asymptotic stability of the endemic equilibrium $E_{\varepsilon}^{*}$ for $\varepsilon>0$ with two specific birth rate functions, $B_{2}(N)=\frac{p}{q+N}$ and $B_{3}(N)=\frac{A}{N}+c$ (see [1, Theorem 4.5]).

Zhao and Zou [5] also studied the global dynamics of (1.3). By a combination of the theory of monotone dynamical systems and uniform persistence theory, they established that the basic reproduction number $R_{0}(\varepsilon)$ works as a threshold parameter which determines the extinction of the disease, even if the disease causes the death of the population $(\varepsilon>0)$. They obtained the following threshold type result.

Theorem A 1 (See Zhao and Zou [5, Theorem 2.1]) Assume that (H1) and (H2) hold.

If $R_{0}(\varepsilon)<1$, then every solution $(I(t, \phi), N(t, \phi))$ of system (1.3) with $\phi \in X_{0}$ satisfies

$$
\lim _{t \rightarrow+\infty} I(t, \phi)=0, \lim _{t \rightarrow+\infty} N(t, \phi)=N^{*}(0) .
$$

If $R_{0}(\varepsilon)>1$, then there is a $\beta>0$ such that every solution $(I(t, \phi), N(t, \phi))$ of system (1.3) with $\phi \in X_{0}$ satisfies

$$
\liminf _{t \rightarrow+\infty} N(t, \phi) \geq \liminf _{t \rightarrow+\infty} I(t, \phi) \geq \beta .
$$

From their result, it is shown that if $R_{0}(\varepsilon)>1$, then the disease persists in the host population. Moreover, they studied the global attractivity of the endemic equilibrium $E_{\varepsilon}^{*}$ by using a perturbation theory. They showed that if $\varepsilon>0$ is small enough, then the endemic equilibrium $E_{\varepsilon}^{*}$ is still globally attractive. 
Theorem A 2 (See Zhao and Zou [5, Theorem 2.2].) Assume that (H1) with $\varepsilon=0$ and (H2) hold. If $R_{0}(0)=\frac{\lambda}{d+\gamma}>1$, then there exists an $\bar{\varepsilon}>0$ such that for any $\varepsilon \in[0, \bar{\varepsilon}]$, system (1.3) admits a endemic equilibrium $E_{\varepsilon}^{*}=\left(I^{*}(\varepsilon), N^{*}(\varepsilon)\right)$ which is globally attractive in $X_{0}$.

However, how to determine $\bar{\varepsilon}$ in Theorem A 2 is still an open problem and the dynamics of (1.3) is not completely understood. Is the endemic equilibrium $E_{\varepsilon}^{*}$ globally asymptotically stable for the large value of $\varepsilon$ ? Such a question also can be found in Zhao and Zou [5] with their numerical simulations.

In this paper, we investigate the global stability of the endemic equilibrium $E_{\varepsilon}^{*}$ by monotone iterative method and establish sufficient conditions for the global stability of the endemic equilibrium $E_{\varepsilon}^{*}$ of (1.3). Our analysis allows us to determine the disease induced death rate $\varepsilon$ for the global stability of the endemic equilibrium $E_{\varepsilon}^{*}$. In fact, we will find $\bar{\varepsilon}$ in Theorem A 2 for $B_{3}(N)=\frac{A}{N}+c$ and $B_{1}(N)=b \mathrm{e}^{-a N}$ in Section 4 .

The organization of this paper is as follows. In the next section, we show the permanence of (1.3) for $R_{0}(\varepsilon)>1$. This implies the disease eventually persists for $R_{0}(\varepsilon)>1$. Indeed, Theorem A 1 by Zhao and Zou [5] also has the same meaning. However, we need to determine the lower and upper bound of the solution explicitly to start the discussion in Section 3. In Section 3, we introduce a set of sequences to estimate the solution by below and above, respectively. By employing a monotone iterative method, Theorems 3.5 and 3.8 are established, dependently on the monotone property of the birth rate function $B(N)$. In Section 4, we study (1.3) with two specific birth rate functions $B_{3}(N)=A / N+c$ and $B_{1}(N)=b \mathrm{e}^{-a N}$ and obtain some global stability results. For each model, the disease induced death rate which guarantees the global stability of the endemic equilibrium is established and this gives a positive answer for the problem proposed by Zhao and Zou [5]. In Section 5, we offer a brief discussion.

\section{Permanence}

In this section, we show that (1.3) is permanent for $R_{0}(\varepsilon)>1$. Indeed, uniformly persistence of the solution is established in Theorem A 1 by Zhao and Zou [5]. However, we need to introduce the following result to derive (2.2). (2.2) will be used as an initial data of the monotone iterative method in the next section.

Theorem 2.1 Assume that (H1) and (H2) hold. If $R_{0}(\varepsilon)>1$, then for any solution of (1.3) in $X_{0}$, it holds that

$$
\left\{\begin{array}{l}
0<\underline{N}_{\varepsilon} \leq \liminf _{t \rightarrow+\infty} N(t) \leq \limsup _{t \rightarrow+\infty} N(t) \leq \bar{N}_{\varepsilon}<+\infty \\
0<\underline{I}_{\varepsilon} \leq \liminf _{t \rightarrow+\infty} I(t) \leq \limsup _{t \rightarrow+\infty} I(t) \leq \bar{I}_{\varepsilon}<+\infty
\end{array}\right.
$$

where

$$
\underline{N}_{\varepsilon}=B^{-1}\left((d+\varepsilon) e^{d_{1} T}\right), \bar{N}_{\varepsilon}=B^{-1}\left(d e^{d_{1} T}\right), \underline{I}_{\varepsilon}=\left(1-\frac{1}{R_{0}(\varepsilon)}\right) \underline{N}_{\varepsilon}, \bar{I}_{\varepsilon}=\left(1-\frac{1}{R_{0}(\varepsilon)}\right) \bar{N}_{\varepsilon} .
$$

PROOF. From the second equation of (1.3),

$$
N^{\prime}(t) \leq B(N(t-T)) N(t-T) \mathrm{e}^{-d_{1} T}-d N(t),
$$

holds. We consider the following auxiliary equation

$$
\bar{N}^{\prime}(t)=B(\bar{N}(t-T)) \bar{N}(t-T) \mathrm{e}^{-d_{1} T}-d \bar{N}(t) .
$$


Since $\lim _{t \rightarrow+\infty} \bar{N}(t)=B^{-1}\left(d \mathrm{e}^{d_{1} T}\right)=N^{*}(0)=\bar{N}_{\varepsilon}$ follows from (H1) and (H2), we see

$$
\limsup _{t \rightarrow+\infty} N(t) \leq \bar{N}_{\varepsilon}
$$

by using the comparison theorem.

From the second equation of (1.3),

$$
N^{\prime}(t) \geq B(N(t-T)) N(t-T) \mathrm{e}^{-d_{1} T}-(d+\varepsilon) N(t),
$$

holds. We consider the following auxiliary equation

$$
\underline{N^{\prime}}(t)=B(\underline{N}(t-T)) \underline{N}(t-T) \mathrm{e}^{-d_{1} T}-(d+\varepsilon) \underline{N}(t) .
$$

Since $\lim _{t \rightarrow+\infty} \underline{N}(t)=B^{-1}\left((d+\varepsilon) \mathrm{e}^{d_{1} T}\right)=\underline{N}_{\varepsilon}$ follows, we see

$$
\liminf _{t \rightarrow+\infty} N(t) \geq \underline{N}_{\varepsilon}
$$

by using the comparison theorem.

For any $\delta_{1}>0$, there exists a $t_{1}$ such that $N(t) \leq \bar{N}_{\varepsilon}+\delta_{1}$ for $t \geq t_{1}$. Then, from the first equation of (1.3) and $R_{0}(\varepsilon)>1$, we obtain that

$$
\begin{aligned}
I^{\prime}(t) & =I(t)\left(\lambda \frac{N(t)-I(t)}{N(t)}-(d+\varepsilon+\gamma)\right)=I(t) \lambda\left(\left(1-\frac{1}{R_{0}(\varepsilon)}\right)-\frac{I(t)}{N(t)}\right) \\
& \leq I(t) \lambda\left(\left(1-\frac{1}{R_{0}(\varepsilon)}\right)-\frac{I(t)}{\bar{N}_{\varepsilon}+\delta_{1}}\right) \text { for } t \geq t_{1} .
\end{aligned}
$$

We consider the following auxiliary equation

$$
\bar{I}^{\prime}(t)=\bar{I}(t) \lambda\left(\left(1-\frac{1}{R_{0}(\varepsilon)}\right)-\frac{\bar{I}(t)}{\bar{N}_{\varepsilon}+\delta_{1}}\right) .
$$

Then, it holds $\lim _{t \rightarrow+\infty} \bar{I}(t)=\left(1-\frac{1}{R_{0}(\varepsilon)}\right)\left(\bar{N}_{\varepsilon}+\delta_{1}\right)$ and hence,

$$
\limsup _{t \rightarrow+\infty} I(t) \leq \bar{I}_{\varepsilon}
$$

follows, since $\delta_{1}$ is arbitrary. Similarly, we also obtain that $\liminf _{t \rightarrow+\infty} I(t) \geq \underline{I}_{\varepsilon}$. Hence the proof is complete.

Remark 2.2 It holds $\underline{N}_{\varepsilon} \leq \bar{N}_{\varepsilon}$, since $B(N)$ is monotone decreasing on $N$ from $(H 1)$.

\section{Global stability of the endemic equilibrium}

In this section, by using monotone iterative techniques, we investigate the global asymptotic stability of the endemic equilibrium $E_{\varepsilon}^{*}$ of (1.3) for $R_{0}(\varepsilon)>1$. We assume $\varepsilon>0$, because our aim is to derive a sufficient condition for the global stability of the endemic equilibrium $E_{\varepsilon}^{*}$ for the case $\varepsilon>0$, that is, the disease causes death of the infective individuals.

We can observe that $G(N)=B(N) N$ is monotone increasing function of $N$ for the birth rate functions $B_{3}(N)=A / N+c$ and $B_{2}(N)=\frac{p}{q+N}$. On the other hand, $G(N)$ is a unimodal function for the birth rate function $B_{1}(N)=b \mathrm{e}^{-a N}$. Hence, to obtain global stability results for these cases, we divide this section into two parts, dependently on the property of $G(N)$. In Section 3.1, we study the global stability when $G(N)$ is monotone increasing. We can apply Theorem 3.5 to 
the case that the birth rate function $B(N)$ satisfies a suitable monotone property, for example, $B_{3}(N)=\frac{A}{N}+c$ and $B_{2}(N)=\frac{p}{q+N}$. In Section 3.2, we study the global stability when $G(N)$ is monotone decreasing on the feasible region of $N$. We apply Theorem 3.8 to that case that $G(N)$ has a unimodal property, for example, $B_{1}(N)=b \mathrm{e}^{-a N}$. We present a graphical representation of $G(N)=B(N) N$ for $B_{1}(N)$ and $B_{3}(N)$ (see Figures 1 and 2).

\subsection{Case: $G(N)$ is a increasing function}

We observe that $G(N)=B(N) N$ is monotone increasing function of $N$ for the birth rate functions $B_{3}(N)=A / N+c$ and $B_{2}(N)=\frac{p}{q+N}$. Thus, in this subsection, we study the case that $G(N)=$ $B(N) N$ is monotone increasing on the feasible region $\left[\underline{N}_{\varepsilon}, \bar{N}_{\varepsilon}\right]$. We assume that

$$
0 \leq G^{\prime}(N) \text { for } N \in\left[\underline{N}_{\varepsilon}, \bar{N}_{\varepsilon}\right] .
$$

Let

$$
H_{1}(N)=B(N) N \mathrm{e}^{-d_{1} T}-d N=G(N) \mathrm{e}^{-d_{1} T}-d N \text { for } N \in\left[\underline{N}_{\varepsilon}, \bar{N}_{\varepsilon}\right] .
$$

If we, further, assume that

$$
0 \leq G^{\prime}(N) \mathrm{e}^{-d_{1} T}<d \text { for } N \in\left[\underline{N}_{\varepsilon}, \bar{N}_{\varepsilon}\right],
$$

then $H_{1}(N)$ is strictly monotone decreasing on $N \in\left[\underline{N}_{\varepsilon}, \bar{N}_{\varepsilon}\right]$ and hence, its inverse function $H_{1}^{-1}(N)$ is well defined and is also strictly monotone decreasing. From (1.4) and (2.2), it holds

$$
\begin{gathered}
H_{1}\left(\underline{N_{\varepsilon}}\right)=(d+\varepsilon) \mathrm{e}^{d_{1} T} \underline{N}_{\varepsilon} \mathrm{e}^{-d_{1} T}-d \underline{N}_{\varepsilon}=\varepsilon \underline{N}_{\varepsilon} \\
H_{1}\left(N^{*}(\varepsilon)\right)=\left(d+\varepsilon\left(1-\frac{1}{R_{0}(\varepsilon)}\right)\right) \mathrm{e}^{d_{1} T} N^{*}(\varepsilon) \mathrm{e}^{-d_{1} T}-d N^{*}(\varepsilon)=\varepsilon\left(1-\frac{1}{R_{0}(\varepsilon)}\right) N^{*}(\varepsilon),
\end{gathered}
$$

and

$$
H_{1}\left(\bar{N}_{\varepsilon}\right)=d \mathrm{e}^{d_{1} T} \bar{N}_{\varepsilon} \mathrm{e}^{-d_{1} T}-d \bar{N}_{\varepsilon}=0 .
$$

Thus,

$$
H_{1}(N):\left[\underline{N}_{\varepsilon}, \bar{N}_{\varepsilon}\right] \rightarrow\left[0, \varepsilon \underline{N}_{\varepsilon}\right], H_{1}^{-1}(N):\left[0, \varepsilon \underline{N}_{\varepsilon}\right] \rightarrow\left[\underline{N}_{\varepsilon}, \bar{N}_{\varepsilon}\right] .
$$

Let us introduce the following four sequences $\left\{\underline{N}_{n}\right\}_{n=0}^{+\infty},\left\{\bar{N}_{n}\right\}_{n=1}^{+\infty},\left\{\underline{I}_{n}\right\}_{n=1}^{+\infty}$ and $\left\{\bar{I}_{n}\right\}_{n=1}^{+\infty}$ such that

$$
\left\{\begin{array}{ll}
\bar{N}_{n}=H_{1}^{-1}\left(\tilde{\varepsilon} \underline{N}_{n-1}\right), & \bar{I}_{n}=\left(1-\frac{1}{R_{0}(\varepsilon)}\right) \bar{N}_{n}, \\
\underline{N}_{n}=H_{1}^{-1}\left(\tilde{\varepsilon} \bar{N}_{n}\right), & \underline{I}_{n}=\left(1-\frac{1}{R_{0}(\varepsilon)}\right) \underline{N}_{n},
\end{array} \text { for } n=1,2,3 \ldots,\right.
$$

where

$$
\underline{N}_{0}=\underline{N}_{\varepsilon}
$$

and

$$
\tilde{\varepsilon}=\varepsilon\left(1-\frac{1}{R_{0}(\varepsilon)}\right)
$$

These sequences will be used as an estimation of the solution. We introduce the following result. 
Lemma 3.1 Assume that (H1), (H2) and (3.2) hold. If

$$
\underline{N}_{\varepsilon} \geq\left(1-\frac{1}{R_{0}(\varepsilon)}\right) \bar{N}_{\varepsilon},
$$

holds, then

$$
0<\underline{N}_{\varepsilon}=\underline{N}_{0} \leq \underline{N}_{n} \leq N^{*}(\varepsilon) \leq \bar{N}_{n} \leq \bar{N}_{\varepsilon}<+\infty, \text { for } n=1,2,3 \ldots
$$

PROOF. By (3.2), $H_{1}(N)$ is monotone decreasing on $N \in\left[\underline{N}_{\varepsilon}, \bar{N}_{\varepsilon}\right]$. From (3.4) and (3.6), it holds

$$
H_{1}\left(\bar{N}_{1}\right)=\tilde{\varepsilon} \underline{N}_{0}=\tilde{\varepsilon} \underline{N}_{\varepsilon} \leq \tilde{\varepsilon} N^{*}(\varepsilon)=H_{1}\left(N^{*}(\varepsilon)\right) .
$$

Since $H_{1}(N)$ is monotone decreasing on $N$, we see

$$
\bar{N}_{1} \geq N^{*}(\varepsilon) \text {. }
$$

On the other hand, from (3.5), it follows

$$
H_{1}\left(\bar{N}_{1}\right)=\tilde{\varepsilon} \underline{N}_{0}=\tilde{\varepsilon} \underline{N}_{\varepsilon} \geq 0=H_{1}\left(\bar{N}_{\varepsilon}\right),
$$

Since $H_{1}(N)$ is monotone decreasing on $N$, we see

$$
\bar{N}_{1} \leq \bar{N}_{\varepsilon} .
$$

From (3.4), (3.6) and (3.9), it holds that

$$
H_{1}\left(\underline{N}_{1}\right)=\tilde{\varepsilon} \bar{N}_{1} \geq \tilde{\varepsilon} N^{*}(\varepsilon)=H_{1}\left(N^{*}(\varepsilon)\right),
$$

Since $H_{1}(N)$ is monotone decreasing on $N$, we see

$$
\underline{N}_{1} \leq N^{*}(\varepsilon) \text {. }
$$

On the other hand, from (3.8), we have

$$
\varepsilon \underline{N}_{\varepsilon} \geq \tilde{\varepsilon} \bar{N}_{\varepsilon}
$$

Then, from (3.3), (3.10) and (3.12), it holds that

$$
H_{1}\left(\underline{N}_{1}\right)=\tilde{\varepsilon} \bar{N}_{1} \leq \tilde{\varepsilon} \bar{N}_{\varepsilon} \leq \varepsilon \underline{N}_{\varepsilon}=H_{1}\left(\underline{N}_{\varepsilon}\right) .
$$

Since $H_{1}(N)$ is monotone decreasing on $N$, we see

$$
\underline{N}_{1} \geq \underline{N}_{\varepsilon}
$$

Consequently, from (3.9), (3.10), (3.11) and (3.13), we obtain

$$
\underline{N}_{\varepsilon}=\underline{N}_{0} \leq \underline{N}_{1} \leq N^{*}(\varepsilon) \leq \bar{N}_{1} \leq \bar{N}_{\varepsilon} .
$$

We show that the conclusion holds by using the mathematical induction. Suppose that $\underline{N}_{\varepsilon} \leq$ $\underline{N}_{n} \leq N^{*}(\varepsilon)$ for some $n \geq 1$. From (3.4), (3.6) and the assumption, we have

$$
H_{1}\left(\bar{N}_{n+1}\right)=\tilde{\varepsilon} \underline{N}_{n} \leq \tilde{\varepsilon} N^{*}(\varepsilon)=H_{1}\left(N^{*}(\varepsilon)\right) .
$$

Since $H_{1}(N)$ is monotone decreasing on $N$, we see

$$
\bar{N}_{n+1} \geq N^{*}(\varepsilon) \text {. }
$$

On the other hand, from the assumption and (3.5), it follows

$$
H_{1}\left(\bar{N}_{n+1}\right)=\tilde{\varepsilon} \underline{N}_{n} \geq \tilde{\varepsilon} \underline{N}_{\varepsilon} \geq 0=H_{1}\left(\bar{N}_{\varepsilon}\right),
$$


Since $H_{1}(N)$ is monotone decreasing on $N$, we see

$$
\bar{N}_{n+1} \leq \bar{N}_{\varepsilon} .
$$

From (3.4), (3.6) and (3.14), we see

$$
H_{1}\left(\underline{N}_{n+1}\right)=\tilde{\varepsilon} \bar{N}_{n+1} \geq \tilde{\varepsilon} N^{*}(\varepsilon)=H_{1}\left(N^{*}(\varepsilon)\right),
$$

Since $H_{1}(N)$ is monotone decreasing on $N$, we see

$$
\underline{N}_{n+1} \leq N^{*}(\varepsilon) \text {. }
$$

From (3.3), (3.12) and (3.15), it holds that

$$
H_{1}\left(\underline{N}_{n+1}\right)=\tilde{\varepsilon} \bar{N}_{n+1} \leq \tilde{\varepsilon} \bar{N}_{\varepsilon} \leq \varepsilon \underline{N}_{\varepsilon}=H_{1}\left(\underline{N}_{\varepsilon}\right),
$$

Since $H_{1}(N)$ is monotone decreasing on $N$, we see

$$
\underline{N}_{n+1} \geq \underline{N}_{\varepsilon} \text {. }
$$

Consequently, from (3.14), (3.15), (3.16) and (3.17), we obtain

$$
\underline{N}_{\varepsilon}=\underline{N}_{0} \leq \underline{N}_{n+1} \leq N^{*}(\varepsilon) \leq \bar{N}_{n+1} \leq \bar{N}_{\varepsilon} .
$$

Thus, we obtain the conclusion by the mathematical induction. Hence, the proof is complete.

Let us consider

$$
F_{1}(\varepsilon)=\left(1-\frac{1}{R_{0}(\varepsilon)}\right) \frac{\bar{N}_{\varepsilon}}{\underline{N}_{\varepsilon}}=\left(1-\frac{1}{R_{0}(\varepsilon)}\right) \frac{B^{-1}\left(d \mathrm{e}^{d_{1} T}\right)}{B^{-1}\left((d+\varepsilon) \mathrm{e}^{d_{1} T}\right)} \text { for } \varepsilon \in(0, \lambda-(d+\gamma)) .
$$

Since

$$
\lim _{\varepsilon \rightarrow+0} F_{1}(\varepsilon)=1-\frac{1}{R_{0}(0)}<1, \lim _{\varepsilon \rightarrow \lambda-(d+\gamma)} F_{1}(\varepsilon)=0
$$

if $R_{0}(0)>R_{0}(\varepsilon)>1$, we see that if $\varepsilon \in(0, \lambda-(d+\gamma))$ is sufficiently close to 0 or $\lambda-(d+\gamma)$, then $F_{1}(\varepsilon)<1$ and hence, (3.8) holds. We define

$$
\Omega_{1}=\left\{\varepsilon \in(0, \lambda-(d+\gamma)) \mid F_{1}(\varepsilon) \leq 1\right\} .
$$

Therefore, if $\varepsilon \in \Omega_{1}$, then $F_{1}(\varepsilon) \leq 1$ which implies that (3.8) holds.

Now we consider the situation where $N_{n}$ and $\bar{N}_{n}$ converge to $N^{*}(\varepsilon)$ as $n \rightarrow+\infty$.

Lemma 3.2 Assume that (H1), (H2) and (3.2) hold. If $\varepsilon \in \Omega_{1}$ defined by (3.19) and

$$
0<\tilde{\varepsilon}<\min _{N \in\left[\underline{N}_{\varepsilon}, \bar{N}_{\varepsilon}\right]}\left\{-H_{1}^{\prime}(N)\right\},
$$

hold, then

$$
\lim _{n \rightarrow+\infty} \underline{N}_{n}=\lim _{n \rightarrow+\infty} \bar{N}_{n}=N^{*}(\varepsilon) .
$$

PROOF. From (3.6), we have the following relation

$$
\left\{\begin{array}{ll}
H_{1}^{-1}\left(\tilde{\varepsilon} \underline{N}_{n}\right) & =\bar{N}_{n+1}, \\
H_{1}^{-1}\left(\tilde{\varepsilon} \bar{N}_{n+1}\right) & =\underline{N}_{n+1},
\end{array} n=0,1,2, \ldots,\right.
$$

which is equivalent to the following

$$
\left\{\begin{array}{ll}
\tilde{\varepsilon} N_{n} & =H_{1}\left(\bar{N}_{n+1}\right), \\
\tilde{\varepsilon} \bar{N}_{n+1} & =H_{1}\left(\underline{N}_{n+1}\right)
\end{array} \text { for } n=0,1,2, \ldots\right.
$$


Let

$$
\left\{\begin{array}{l}
\underline{l}_{n}=N^{*}(\varepsilon)-\underline{N}_{n}, n=0,1,2, \ldots, \\
\bar{l}_{n}=\bar{N}_{n}-N^{*}(\varepsilon), n=1,2,3, \ldots
\end{array}\right.
$$

Then we have $\underline{l}_{n} \geq 0$ and $\bar{l}_{n} \geq 0$ by Lemma 3.1. There exist $\underline{\eta}_{n} \in\left[\underline{N}_{n}, N^{*}(\varepsilon)\right], n=0,1,2, \ldots$, and $\bar{\eta}_{n} \in\left[N^{*}(\varepsilon), \bar{N}_{n}\right], n=1,2,3 \ldots$, such that

$$
\begin{cases}\tilde{\varepsilon} \underline{l}_{n} & =H_{1}\left(N^{*}(\varepsilon)\right)-H_{1}\left(\bar{N}_{n+1}\right)=-H_{1}^{\prime}\left(\bar{\eta}_{n+1}\right) \bar{l}_{n+1}, \\ \tilde{\varepsilon} \tilde{l}_{n+1} & =H_{1}\left(\underline{N}_{n+1}\right)-H_{1}\left(N^{*}(\varepsilon)\right)=-H_{1}^{\prime}\left(\underline{\eta}_{n+1}\right) \underline{l}_{n+1},\end{cases}
$$

by (3.4), (3.21) and the mean value theorem. Then, from (3.22), we obtain

$$
\underline{l}_{n+1}=\frac{\tilde{\varepsilon}}{-H_{1}^{\prime}\left(\underline{\eta}_{n+1}\right)} \bar{l}_{n+1}=\left(\frac{\tilde{\varepsilon}}{-H_{1}^{\prime}\left(\underline{\eta}_{n+1}\right)}\right)\left(\frac{\tilde{\varepsilon}}{-H_{1}^{\prime}\left(\bar{\eta}_{n+1}\right)}\right) \underline{l}_{n} .
$$

Moreover,

$$
\underline{l}_{n+1} \leq\left(\frac{\tilde{\varepsilon}}{\min _{N \in\left[\underline{N}_{\varepsilon}, \bar{N}_{\varepsilon}\right]}\left\{-H_{1}^{\prime}(N)\right\}}\right)^{2} \underline{l}_{n}<\underline{l}_{n},
$$

follows by (3.20). Thus, $\lim _{n \rightarrow+\infty} \underline{l}_{n}=0$ follows and hence, we obtain $\lim _{n \rightarrow+\infty} \underline{N}_{n}=N^{*}(\varepsilon)$. Similarly, $\lim _{n \rightarrow+\infty} \bar{N}_{n}=N^{*}(\varepsilon)$ holds. Hence, the proof is complete.

Let us consider the following auxiliary equation with a fixed $n$, before giving the main result in this subsection.

$$
\tilde{N}^{\prime}(t)=G(\tilde{N}(t-T)) \mathrm{e}^{-d_{1} T}-d \tilde{N}(t)-\tilde{\varepsilon} \underline{N}_{n},
$$

with initial conditions $\tilde{N}(\theta)=\phi_{\tilde{N}}(\theta)$ for $\theta \in[-T, 0]$ where $\phi_{\tilde{N}} \in C\left([-T, 0],\left[\underline{N}_{\varepsilon}, \bar{N}_{\varepsilon}\right]\right)$.

For (3.23), we have the following lemma.

Lemma 3.3 Assume that (H1), (H2) and (3.2) hold. Then,

$$
\underline{N}_{\varepsilon} \leq \liminf _{t \rightarrow+\infty} \tilde{N}(t) \leq \limsup _{t \rightarrow+\infty} \tilde{N}(t) \leq \bar{N}_{\varepsilon}
$$

for (3.23).

PROOF. Suppose that there exists a $t_{1}$ such that $\tilde{N}\left(t_{1}\right)=\bar{N}_{\varepsilon}$ and $\tilde{N}(t)<\bar{N}_{\varepsilon}$ for $t<t_{1}$. Then we see

$$
\tilde{N}^{\prime}\left(t_{1}\right)=G\left(\tilde{N}\left(t_{1}-T\right)\right) \mathrm{e}^{-d_{1} T}-d \bar{N}_{\varepsilon}-\tilde{\varepsilon} \underline{N}_{n} .
$$

From (3.2), $G(N)$ is monotone increasing and hence, it follows

$$
\begin{aligned}
\tilde{N}^{\prime}\left(t_{1}\right) & \leq G\left(\bar{N}_{\varepsilon}\right) \mathrm{e}^{-d_{1} T}-d \bar{N}_{\varepsilon}-\tilde{\varepsilon} \underline{N}_{n} \\
& =H_{1}\left(\bar{N}_{\varepsilon}\right)-\tilde{\varepsilon} \underline{N}_{n} \\
& =-\tilde{\varepsilon} \underline{N}_{n}<0,
\end{aligned}
$$

by (3.5). This implies that $\limsup _{t \rightarrow+\infty} \tilde{N}(t) \leq \bar{N}_{\varepsilon}$.

On the other hand, suppose that there exists a $t_{2}$ such that $\tilde{N}\left(t_{2}\right)=\underline{N}_{\varepsilon}$ and $\tilde{N}(t)>\underline{N}_{\varepsilon}$ for $t<t_{2}$. Then we see

$$
\tilde{N}^{\prime}\left(t_{2}\right)=G\left(\tilde{N}\left(t_{2}-T\right)\right) \mathrm{e}^{-d_{1} T}-d \underline{N}_{\varepsilon}-\tilde{\varepsilon} \underline{N}_{n} .
$$


From (3.2), $G(N)$ is monotone increasing and hence, it follows

$$
\begin{aligned}
\tilde{N}^{\prime}\left(t_{2}\right) & \geq G\left(\underline{N}_{\varepsilon}\right) \mathrm{e}^{-d_{1} T}-d \underline{N}_{\varepsilon}-\tilde{\varepsilon} \underline{N}_{n} \\
& =H_{1}\left(\underline{N}_{\varepsilon}\right)-\tilde{\varepsilon} \underline{N}_{n} \\
& =\varepsilon \underline{N}_{\varepsilon}-\tilde{\varepsilon} \underline{N}_{n} \\
& \geq(\varepsilon-\tilde{\varepsilon}) \underline{N}_{\varepsilon}>0,
\end{aligned}
$$

by (3.3). This implies that $\underline{N}_{\varepsilon} \leq \liminf _{t \rightarrow+\infty} \tilde{N}(t)$. Thus we obtain the conclusion.

(3.23) has a positive equilibrium $\tilde{N}^{*}$ which satisfies

$$
G\left(\tilde{N}^{*}\right) \mathrm{e}^{-d_{1} T}-d \tilde{N}^{*}-\tilde{\varepsilon} \underline{N}_{n}=H_{1}\left(\tilde{N}^{*}\right)-\tilde{\varepsilon} \underline{N}_{n}=0 .
$$

Then,

$$
\tilde{N}^{*}=H_{1}^{-1}\left(\tilde{\varepsilon} \underline{N}_{n}\right)=\bar{N}_{n+1} .
$$

From the following lemma, every solution converges to the positive equilibrium $\tilde{N}^{*}$.

Lemma 3.4 Assume that (H1), (H2) and (3.2) hold. Then,

$$
\lim _{t \rightarrow+\infty} \tilde{N}(t)=\tilde{N}^{*}
$$

for (3.23).

PROOF. There exists a positive sequence $\left\{t_{h}\right\}_{h=1}^{+\infty}$ such that $\lim _{h \rightarrow+\infty} t_{h}=+\infty$ and

$$
\tilde{N}^{\prime}\left(t_{h}\right) \geq 0, \tilde{N}(t) \leq \tilde{N}\left(t_{h}\right), \text { for } t \leq t_{h}, h=1,2, \ldots, \text { and } \lim _{h \rightarrow+\infty} \tilde{N}\left(t_{h}\right)=\limsup _{t \rightarrow+\infty} \tilde{N}(t) .
$$

Then, we have that

$$
0 \leq \tilde{N}^{\prime}\left(t_{h}\right)=G\left(\tilde{N}\left(t_{h}-T\right)\right) \mathrm{e}^{-d_{1} T}-d \tilde{N}\left(t_{h}\right)-\tilde{\varepsilon} \underline{N}_{n} .
$$

Since $\tilde{N}\left(t_{h}-T\right) \leq \tilde{N}\left(t_{h}\right)$ and, from (3.2), $G(N)$ is monotone increasing of $N$, we have $G\left(\tilde{N}\left(t_{h}-\right.\right.$ $T)) \leq G\left(\tilde{N}\left(t_{h}\right)\right)$. Then, it holds that

$$
0 \leq G\left(\tilde{N}\left(t_{h}\right)\right) \mathrm{e}^{-d_{1} T}-d \tilde{N}\left(t_{h}\right)-\tilde{\varepsilon} \underline{N}_{n}=H_{1}\left(\tilde{N}\left(t_{h}\right)\right)-\tilde{\varepsilon} \underline{N}_{n} .
$$

We have $\bar{N}_{n+1}=H_{1}^{-1}\left(\tilde{\varepsilon} \underline{N}_{n}\right)$ from (3.6) and $H_{1}(N)$ is decreasing on $N$ by (3.2). Then $\tilde{N}\left(t_{h}\right) \leq$ $\bar{N}_{n+1}$ follows. Thus, $\lim \sup _{t \rightarrow+\infty} \tilde{N}(t) \leq \tilde{N}^{*}=H_{1}^{-1}\left(\tilde{\varepsilon} \underline{N}_{n}\right)=\bar{N}_{n+1}$.

Similarly, we obtain $\liminf _{t \rightarrow+\infty} \tilde{N}(t) \geq \tilde{N}^{*}=H_{1}^{-1}\left(\tilde{\varepsilon} \underline{N}_{n}\right)=\bar{N}_{n+1}$. Consequently, $\lim _{t \rightarrow+\infty} \tilde{N}(t)=$ $\tilde{N}^{*}$ holds and the proof is complete.

We establish the following result.

Theorem 3.5 Assume that (H1) and (H2) hold. If $R_{0}(\varepsilon)>1$ then (1.3) admits an endemic equilibrium $E_{\varepsilon}^{*}=\left(I^{*}(\varepsilon), N^{*}(\varepsilon)\right)$. In addition, assume that (3.2). Then, for any $\varepsilon \in \Omega_{1}$ defined by (3.19) such that

$$
0<\varepsilon\left(1-\frac{d+\varepsilon+\gamma}{\lambda}\right)<-\max _{N \in\left[\underline{N}_{\varepsilon}, \bar{N}_{\varepsilon}\right]}\left\{G^{\prime}(N) e^{-d_{1} T}\right\}+d
$$

the endemic equilibrium $E_{\varepsilon}^{*}=\left(I^{*}(\varepsilon), N^{*}(\varepsilon)\right)$ is globally asymptotically stable in $X_{0}$. 
PROOF. At first, by Theorem 2.1, we have $\liminf _{t \rightarrow+\infty} N(t) \geq \underline{N}_{0}$ and $\liminf _{t \rightarrow+\infty} I(t) \geq \underline{I}_{0}$, if $R_{0}(\varepsilon)>1$. Then we obtain the following limiting equation

$$
N^{\prime}(t) \leq G(N(t-T)) \mathrm{e}^{-d_{1} T}-d N(t)-\varepsilon \underline{I}_{0}=G(N(t-T)) \mathrm{e}^{-d_{1} T}-d N(t)-\tilde{\varepsilon} \underline{N}_{0} .
$$

By Lemma 3.4 and the comparison theorem, we obtain

$$
\limsup _{t \rightarrow+\infty} N(t) \leq H_{1}^{-1}\left(\tilde{\varepsilon} \underline{N}_{0}\right)=\bar{N}_{1} .
$$

Similar to the proof of Theorem 2.1, we see $\lim \sup _{t \rightarrow+\infty} I(t) \leq \bar{I}_{1}$.

Then, we obtain the following limiting equation

$$
N^{\prime}(t) \geq G(N(t-T)) \mathrm{e}^{-d_{1} T}-d N(t)-\varepsilon \bar{I}_{1}=G(N(t-T)) \mathrm{e}^{-d_{1} T}-d N(t)-\tilde{\varepsilon} \bar{N}_{1} .
$$

By Lemma 3.4 and the comparison theorem, we obtain

$$
\liminf _{t \rightarrow+\infty} N(t) \geq H_{1}^{-1}\left(\tilde{\varepsilon} \bar{N}_{1}\right)=\underline{N}_{1} .
$$

Similar to the proof of Theorem 2.1, we see $\liminf _{t \rightarrow+\infty} I(t) \geq \underline{I}_{1}$.

Repeating the above arguments, we obtain

$$
\underline{N}_{n} \leq \liminf _{t \rightarrow+\infty} N(t) \leq \limsup _{t \rightarrow+\infty} N(t) \leq \bar{N}_{n}, n=1,2,3 \ldots
$$

We see that (3.20) in Lemma 3.2 holds from (3.24). Then, by letting $n \rightarrow+\infty$, it follows

$$
N^{*}(\varepsilon) \leq \liminf _{t \rightarrow+\infty} N(t) \leq \limsup _{t \rightarrow+\infty} N(t) \leq N^{*}(\varepsilon),
$$

which implies the conclusion of this theorem. The proof is complete.

\subsection{Case: $G(N)$ is a unimodal function}

In this subsection, we study the case that $G(N)=B(N) N$ is monotone decreasing on the feasible region $N \in\left[\underline{N}_{\varepsilon}, \bar{N}_{\varepsilon}\right]$. Therefore, we assume that

$$
0 \geq G^{\prime}(N), \text { for any } N \in\left[\underline{N}_{\varepsilon}, \bar{N}_{\varepsilon}\right]
$$

Let

$$
H_{2}(N)=B(N) N \mathrm{e}^{-d_{1} T}-\tilde{\varepsilon} N=G(N) \mathrm{e}^{-d_{1} T}-\tilde{\varepsilon} N \text { for } N \in\left[\underline{N}_{\varepsilon}, \bar{N}_{\varepsilon}\right] .
$$

then $H_{2}(N)$ is strictly monotone decreasing on $N \in\left[\underline{N}_{\varepsilon}, \bar{N}_{\varepsilon}\right]$ from (3.25). From (1.4) and (2.2), it holds

and

$$
\begin{gathered}
H_{2}\left(\underline{N}_{\varepsilon}\right)=(d+\varepsilon) \mathrm{e}^{d_{1} T} \underline{N}_{\varepsilon} \mathrm{e}^{-d_{1} T}-\tilde{\varepsilon} N_{\varepsilon}=(d+\varepsilon-\tilde{\varepsilon}) \underline{N}_{\varepsilon}, \\
H_{2}\left(N^{*}(\varepsilon)\right)=(d+\tilde{\varepsilon}) \mathrm{e}^{d_{1} T} N^{*}(\varepsilon) \mathrm{e}^{-d_{1} T}-\tilde{\varepsilon} N^{*}(\varepsilon)=d N^{*}(\varepsilon),
\end{gathered}
$$

$$
H_{2}\left(\bar{N}_{\varepsilon}\right)=d \mathrm{e}^{d_{1} T} \bar{N}_{\varepsilon} \mathrm{e}^{-d_{1} T}-\tilde{\varepsilon} \bar{N}_{\varepsilon}=(d-\tilde{\varepsilon}) \bar{N}_{\varepsilon} .
$$

Now we introduce the following four sequences $\left\{\underline{N}_{n}\right\}_{n=0}^{+\infty},\left\{\bar{N}_{n}\right\}_{n=1}^{+\infty},\left\{\underline{I}_{n}\right\}_{n=1}^{+\infty}$ and $\left\{\bar{I}_{n}\right\}_{n=1}^{+\infty}$ such that

with

$$
\begin{cases}\bar{N}_{n}=\frac{1}{d} H_{2}\left(\underline{N}_{n-1}\right), & \bar{I}_{n}=\left(1-\frac{1}{R_{0}(\varepsilon)}\right) \bar{N}_{n}, \\ \underline{N}_{n}=\frac{1}{d} H_{2}\left(\bar{N}_{n}\right), & \underline{I}_{n}=\left(1-\frac{1}{R_{0}(\varepsilon)}\right) \underline{N}_{n},\end{cases}
$$

$$
\underline{N}_{0}=\underline{N}_{\varepsilon}
$$

These sequences will be used as an estimation of the solution. We introduce the following result. 
Lemma 3.6 Assume that (H1), (H2) and (3.25) hold. If $\tilde{\varepsilon}<d$ and

$$
d \underline{N}_{\varepsilon} \leq(d-\tilde{\varepsilon}) \bar{N}_{\varepsilon},(d+\varepsilon-\tilde{\varepsilon}) \underline{N}_{\varepsilon} \leq d \bar{N}_{\varepsilon},
$$

hold, then,

$$
0<\underline{N}_{\varepsilon}=\underline{N}_{0} \leq \underline{N}_{n} \leq N^{*}(\varepsilon) \leq \bar{N}_{n} \leq \bar{N}_{\varepsilon}<+\infty, \text { for } n=1,2,3 \ldots
$$

PROOF. By (3.25), $H_{2}(N)$ is monotone decreasing on $N \in\left[\underline{N}_{\varepsilon}, \bar{N}_{\varepsilon}\right]$. From (3.28) and (3.30), it holds

$$
d \bar{N}_{1}=H_{2}\left(\underline{N}_{0}\right)=H_{2}\left(\underline{N}_{\varepsilon}\right) \geq H_{2}\left(N^{*}(\varepsilon)\right)=d N^{*}(\varepsilon),
$$

since $H_{2}(N)$ is monotone decreasing on $N$. Then,

$$
\bar{N}_{1} \geq N^{*}(\varepsilon),
$$

follows. On the other hand, from (3.31), we have

$$
(d+\varepsilon-\tilde{\varepsilon}) \underline{N}_{\varepsilon} \leq d \bar{N}_{\varepsilon} .
$$

Then, from (3.27), it follows that

$$
d \bar{N}_{1}=H_{2}\left(\underline{N}_{0}\right)=H_{2}\left(\underline{N}_{\varepsilon}\right)=(d+\varepsilon-\tilde{\varepsilon}) \underline{N}_{\varepsilon} \leq d \bar{N}_{\varepsilon},
$$

and hence, we obtain

$$
\bar{N}_{1} \leq \bar{N}_{\varepsilon}
$$

From (3.28), (3.30) and (3.32), it holds

$$
d \underline{N}_{1}=H_{2}\left(\bar{N}_{1}\right) \leq H_{2}\left(N^{*}(\varepsilon)\right)=d N^{*}(\varepsilon),
$$

since $\mathrm{H}_{2}(\mathrm{~N})$ is monotone decreasing on $N$. Then, we see

$$
\underline{N}_{1} \leq N^{*}(\varepsilon) \text {. }
$$

On the other hand, from (3.31), we have

$$
(d-\tilde{\varepsilon}) \bar{N}_{\varepsilon} \geq d \underline{N}_{\varepsilon}
$$

Then, from (3.34) and (3.29), it holds

$$
d \underline{N}_{1}=H_{2}\left(\bar{N}_{1}\right) \geq H_{2}\left(\bar{N}_{\varepsilon}\right)=(d-\tilde{\varepsilon}) \bar{N}_{\varepsilon} \geq d \underline{N}_{\varepsilon},
$$

since $\mathrm{H}_{2}(\mathrm{~N})$ is monotone decreasing on $N$. Then, we see

$$
\underline{N}_{1} \geq \underline{N}_{\varepsilon} \text {. }
$$

Consequently, from (3.32), (3.34), (3.35) and (3.37), it holds

$$
\underline{N}_{\varepsilon} \leq \underline{N}_{1} \leq N^{*}(\varepsilon) \leq \bar{N}_{1} \leq \bar{N}_{\varepsilon}
$$

We show that the conclusion holds by using the mathematical induction. Suppose that $\underline{N}_{\varepsilon} \leq$ $\underline{N}_{n} \leq N^{*}(\varepsilon)$ for some $n \geq 1$. From (3.28) and (3.30), it holds

$$
d \bar{N}_{n+1}=H_{2}\left(\underline{N}_{n}\right) \geq H_{2}\left(N^{*}(\varepsilon)\right)=d N^{*}(\varepsilon),
$$

since $H_{2}(N)$ is monotone decreasing on $N$. Then, we see

$$
\bar{N}_{n+1} \geq N^{*}(\varepsilon)
$$

Since we have (3.33), from the assumption and (3.27), we see

$$
d \bar{N}_{n+1}=H_{2}\left(\underline{N}_{n}\right) \leq H_{2}\left(\underline{N}_{\varepsilon}\right)=(d+\varepsilon-\tilde{\varepsilon}) \underline{N}_{\varepsilon} \leq d \bar{N}_{\varepsilon},
$$


Then, we see

$$
\bar{N}_{n+1} \leq \bar{N}_{\varepsilon}
$$

From (3.28), (3.30) and (3.38), it holds

$$
d \underline{N}_{n+1}=H_{2}\left(\bar{N}_{n+1}\right) \leq H_{2}\left(N^{*}(\varepsilon)\right)=d N^{*}(\varepsilon),
$$

since $\mathrm{H}_{2}(\mathrm{~N})$ is monotone decreasing on $N$. Then, we see

$$
\underline{N}_{n+1} \leq N^{*}(\varepsilon) \text {. }
$$

Since we have (3.36), from (3.29) and (3.39), it holds

$$
d \underline{N}_{n+1}=H_{2}\left(\bar{N}_{n+1}\right) \geq H_{2}\left(\bar{N}_{\varepsilon}\right)=(d-\tilde{\varepsilon}) \bar{N}_{\varepsilon} \geq d \underline{N}_{\varepsilon},
$$

since $\mathrm{H}_{2}(\mathrm{~N})$ is monotone decreasing on $N$. Then, we see

$$
\underline{N}_{n+1} \geq \underline{N}_{\varepsilon}
$$

Consequently, from (3.38), (3.39), (3.40) and (3.41), we obtain

$$
\underline{N}_{\varepsilon}=\underline{N}_{0} \leq \underline{N}_{n+1} \leq N^{*}(\varepsilon) \leq \bar{N}_{n+1} \leq \bar{N}_{\varepsilon}
$$

Thus, we obtain the conclusion by the mathematical induction. Hence, the proof is complete.

Now we consider

$$
\left\{\begin{array}{l}
F_{2}(\varepsilon)=\frac{d \underline{N}_{\varepsilon}}{(d-\tilde{\varepsilon}) \bar{N}_{\varepsilon}}=\frac{d B^{-1}\left((d+\varepsilon) \mathrm{e}^{d_{1} T}\right)}{(d-\tilde{\varepsilon}) B^{-1}\left(d \mathrm{e}^{d_{1} T}\right)}, \\
F_{3}(\varepsilon)=\frac{(d+\varepsilon-\tilde{\varepsilon}) \underline{N}_{\varepsilon}}{d \bar{N}_{\varepsilon}}=\frac{(d+\varepsilon-\tilde{\varepsilon}) B^{-1}\left((d+\varepsilon) \mathrm{e}^{d_{1} T}\right)}{d B^{-1}\left(d \mathrm{e}^{d_{1} T}\right)},
\end{array}\right.
$$

under the condition $\tilde{\varepsilon}<d$. We see from (3.7)

$$
\lim _{\varepsilon \rightarrow+0} F_{j}(\varepsilon)=1, j=2,3
$$

if $R_{0}(0)>R_{0}(\varepsilon)>1$. We assume

- (F1) If $\varepsilon$ is sufficiently small, then $F_{j}(\varepsilon) \leq 1$ for $j=2,3$,

and define

$$
\Omega_{2}=\left\{\varepsilon \in(0, \lambda-(d+\gamma)) \mid F_{j}(\varepsilon) \leq 1, j=2,3\right\} .
$$

We see that if $\varepsilon \in \Omega_{2}$ then, $F_{j}(\varepsilon) \leq 1, j=2,3$ which implies that (3.31) holds. In Section 4, we discuss on a sufficient condition which ensure (F1) for $B_{1}(N)=b \mathrm{e}^{-a N}$.

Now we consider the situation where $\underline{N}_{n}$ and $\bar{N}_{n}$ converge to $N^{*}(\varepsilon)$ as $n \rightarrow+\infty$.

Lemma 3.7 Assume that (H1), (H2), (3.25) and (F1) hold. If $\varepsilon \in \Omega_{2}$ defined by (3.43) and

$$
\max _{N \in\left[\underline{N}_{\varepsilon}, \bar{N}_{\varepsilon}\right]}\left\{-H_{2}^{\prime}(N)\right\}<d,
$$

hold, then,

$$
\lim _{n \rightarrow+\infty} \underline{N}_{n}=\lim _{n \rightarrow+\infty} \bar{N}_{n}=N^{*}(\varepsilon)
$$


PROOF. Let

$$
\left\{\begin{array}{l}
\underline{l}_{n}=N^{*}(\varepsilon)-\underline{N}_{n}, n=0,1,2, \ldots, \\
\bar{l}_{n}=\bar{N}_{n}-N^{*}(\varepsilon), n=1,2,3, \ldots
\end{array}\right.
$$

We have $\underline{l}_{n} \geq 0$ and $\bar{l}_{n} \geq 0$ by Lemma 3.6. There exist $\bar{\eta}_{n} \in\left[N^{*}(\varepsilon), \bar{N}_{n}\right]$ and $\underline{\eta}_{n} \in\left[\underline{N}_{n}, N^{*}(\varepsilon)\right]$, $n=1,2,3 \ldots$ such that

$$
\left\{\begin{array}{l}
d \bar{l}_{n+1}=H_{2}\left(\underline{N}_{n}\right)-H_{2}\left(N^{*}(\varepsilon)\right)=-H_{2}^{\prime}\left(\underline{\eta}_{n}\right) \underline{l}_{n}, n=0,1,2, \ldots \\
d \underline{l}_{n+1}=H_{2}\left(N^{*}(\varepsilon)\right)-H_{2}\left(\bar{N}_{n+1}\right)=-H_{2}^{\prime}\left(\underline{\eta}_{n+1}\right) \bar{l}_{n+1}, n=1,2,3, \ldots,
\end{array}\right.
$$

by (3.28), (3.30) and the mean value theorem. Then, from (3.45) we obtain

$$
\bar{l}_{n+1}=\frac{-H_{2}^{\prime}\left(\underline{\eta}_{n}\right)}{d} \underline{l}_{n}=\left(\frac{-H_{2}^{\prime}\left(\underline{\eta}_{n}\right)}{d}\right)\left(\frac{-H_{2}^{\prime}\left(\underline{\eta}_{n+1}\right)}{d}\right) \bar{l}_{n} .
$$

Moreover,

$$
\bar{l}_{n+1} \leq\left(\frac{\max _{N \in\left[\underline{N}_{\varepsilon}, \bar{N}_{\varepsilon}\right]}\left\{-H_{2}^{\prime}(N)\right\}}{d}\right)^{2} \bar{l}_{n}<\bar{l}_{n},
$$

follows by (3.44). Thus, $\lim _{n \rightarrow+\infty} \bar{l}_{n}=0$ follows and hence, we obtain $\lim _{n \rightarrow+\infty} \bar{N}_{n}=N^{*}(\varepsilon)$. Similarly, $\lim _{n \rightarrow+\infty} \underline{N}_{n}=N^{*}(\varepsilon)$ holds. Hence, the proof is complete.

Then, we give the following result.

Theorem 3.8 Assume that $(H 1)$ and $(H 2)$ hold. If $R_{0}(\varepsilon)>1$ then (1.3) admits an endemic equilibrium $E_{\varepsilon}^{*}=\left(I^{*}(\varepsilon), N^{*}(\varepsilon)\right)$. In addition, assume that (3.25) and (F1) hold. Then, for any $\varepsilon \in \Omega_{2}$ defined by (3.43) such that

$$
0<\varepsilon\left(1-\frac{d+\varepsilon+\gamma}{\lambda}\right)<\min _{N \in\left[\underline{N}_{\varepsilon}, \bar{N}_{\varepsilon}\right]}\left\{G^{\prime}(N) \mathrm{e}^{-d_{1} T}\right\}+d,
$$

the endemic equilibrium $E_{\varepsilon}^{*}=\left(I^{*}(\varepsilon), N^{*}(\varepsilon)\right)$ is globally asymptotically stable in $X_{0}$.

PROOF. At first, by Theorem 2.1, we have that $\liminf _{t \rightarrow+\infty} N(t) \geq \underline{N}_{0}$ and $\liminf _{t \rightarrow+\infty} I(t) \geq \underline{I}_{0}$. Since $G(N)$ is monotone decreasing on $N \in\left[\underline{N}_{\varepsilon}, \bar{N}_{\varepsilon}\right]$ under the condition (3.25), we obtain the following limiting equation

$$
\begin{aligned}
N^{\prime}(t) & \leq G\left(\underline{N}_{0}\right) \mathrm{e}^{-d_{1} T}-d N(t)-\varepsilon \underline{I}_{0} \\
& =G\left(\underline{N}_{0}\right) \mathrm{e}^{-d_{1} T}-d N(t)-\tilde{\varepsilon} \underline{N}_{0}=H_{2}\left(\underline{N}_{0}\right)-d N(t) .
\end{aligned}
$$

By the comparison theorem, we obtain

$$
\limsup _{t \rightarrow+\infty} N(t) \leq \frac{1}{d} H_{2}\left(\underline{N}_{0}\right)=\bar{N}_{1} .
$$

Similar to the proof of Theorem 2.1, we see limsup $\operatorname{su}_{t \rightarrow+\infty} I(t) \leq \bar{I}_{1}$ follows.

Since $G(N)$ is monotone decreasing on $N \in\left[\underline{N}_{\varepsilon}, \bar{N}_{\varepsilon}\right]$ under the condition (3.25), we obtain the following limiting equation

$$
\begin{aligned}
N^{\prime}(t) & \geq G\left(\bar{N}_{1}\right) \mathrm{e}^{-d_{1} T}-d N(t)-\varepsilon \bar{I}_{1} \\
& =G\left(\bar{N}_{1}\right) \mathrm{e}^{-d_{1} T}-d N(t)-\tilde{\varepsilon} \bar{N}_{1}=H_{2}\left(\bar{N}_{1}\right)-d N(t) .
\end{aligned}
$$




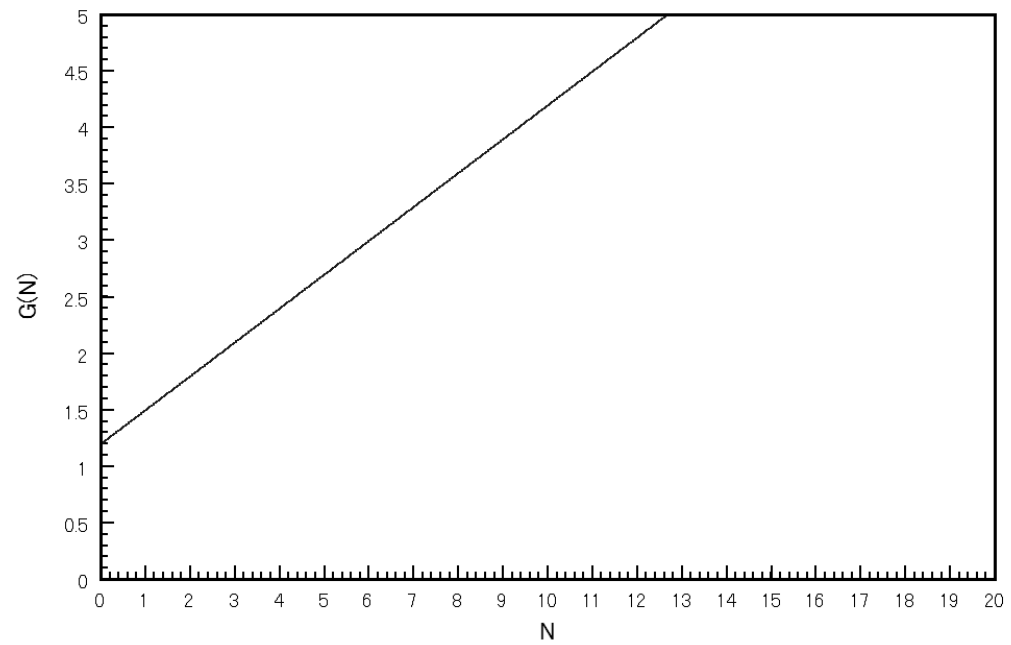

Fig. 1. Graph trajectory of $G(N)=B(N) N=A+c N$ with $A=1.2$ and $c=0.3$

By the comparison theorem, we obtain

$$
\liminf _{t \rightarrow+\infty} N(t) \geq \frac{1}{d} H_{2}\left(\bar{N}_{1}\right)=\underline{N}_{1}
$$

Similar to the proof of Theorem 2.1, we see $\liminf _{t \rightarrow+\infty} I(t) \geq \underline{I}_{1}$.

Repeating the above arguments, we obtain

$$
\underline{N}_{n} \leq \liminf _{t \rightarrow+\infty} N(t) \leq \limsup _{t \rightarrow+\infty} N(t) \leq \bar{N}_{n}, n=1,2,3 \ldots
$$

We see that (3.44) in Lemma 3.7 holds from (3.46). Then, by letting $n \rightarrow+\infty$, it follows

$$
N^{*}(\varepsilon) \leq \liminf _{t \rightarrow+\infty} N(t) \leq \limsup _{t \rightarrow+\infty} N(t) \leq N^{*}(\varepsilon),
$$

which implies the conclusion of this theorem. The proof is complete.

From Theorems 3.5 and 3.8, we can determine $\varepsilon$ which guarantees the global stability of the endemic equilibrium $E_{\varepsilon}^{*}$, respectively. This allows us to find $\bar{\varepsilon}$ in Theorem A 2 by Zhao and Zou [5].

\section{Applications}

In this section, we consider (1.3) with two specific birth rate functions $B_{3}(N)=\frac{A}{N}+c$ and $B_{1}(N)=b \mathrm{e}^{-a N}$. For the reader, we illustrate the graph of $G(N)=B(N) N$ for these birth rate functions, respectively (Figures 1 and 2). For the case $B_{3}(N)=\frac{A}{N}+c$ in (1.3), we establish Theorem 4.1 from Theorem 3.5, because $G(N)=A+c N$ is increasing of $N$. For the case $B_{1}(N)=$ $b \mathrm{e}^{-a N}$ in (1.3), $G(N)=b \mathrm{e}^{-a N} N$ is increasing on $N \in\left(0, \frac{1}{a}\right]$ and decreasing on $N \in\left[\frac{1}{a},+\infty\right)$. We obtain two global stability results, Theorems 4.3 and 4.4, from Theorems 3.5 and 3.8, respectively. 


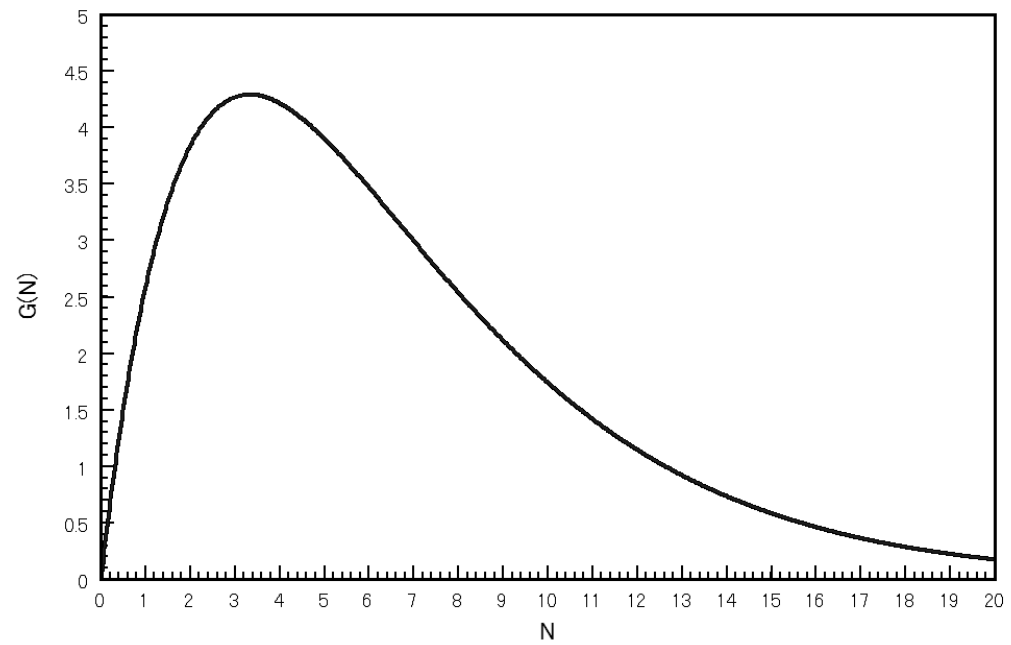

Fig. 2. Graph trajectory of $G(N)=B(N) N=b \mathrm{e}^{-a N} N$ with $b=3.5$ and $a=0.3$

4.1. Case: $B_{3}(N)=A / N+c$

In this subsection, we study (1.3) with $B_{3}(N)=\frac{A}{N}+c$. We consider the following system

$$
\left\{\begin{array}{l}
I^{\prime}(t)=\lambda(N(t)-I(t)) \frac{I(t)}{N(t)}-(d+\varepsilon+\gamma) I(t), \\
N^{\prime}(t)=[A+c N(t-T)] \mathrm{e}^{-d_{1} T}-d N(t)-\varepsilon I(t) .
\end{array}\right.
$$

For (H1), we assume

$$
\lim _{N \rightarrow+0} B_{3}(N)=+\infty>(d+\varepsilon) \mathrm{e}^{d_{1} T} \geq d \mathrm{e}^{d_{1} T}>\lim _{N \rightarrow+\infty} B_{3}(N)=c>0 .
$$

Then, (4.1) has the disease free equilibrium $E_{0}=\left(0, B^{-1}\left(d \mathrm{e}^{d_{1} T}\right)\right)$ and the endemic equilibrium $E_{\varepsilon}^{*}=\left(I^{*}(\varepsilon), N^{*}(\varepsilon)\right)$ if $R_{0}(\varepsilon)=\frac{\lambda}{d+\varepsilon+\gamma}>1$. Since $G^{\prime}(N)=c>0$, (H2) also holds.

From Theorem 2.1, it holds

$$
0<\underline{N_{\varepsilon}} \leq \liminf _{t \rightarrow+\infty} N(t) \leq \limsup _{t \rightarrow+\infty} N(t) \leq \bar{N}_{\varepsilon}<+\infty,
$$

where

$$
\underline{N}_{\varepsilon}=\frac{A}{(d+\varepsilon) \mathrm{e}^{d_{1} T}-c}, \bar{N}_{\varepsilon}=\frac{A}{{d \mathrm{e}^{d_{1} T}-c}},
$$

if $R_{0}(\varepsilon)>1$.

Then, we establish the following result which is obtained from Theorem 3.5.

Theorem 4.1 Assume that (4.2) and $R_{0}(\varepsilon)>1$ then (4.1) admits an endemic equilibrium $E_{\varepsilon}^{*}=$ $\left(I^{*}(\varepsilon), N^{*}(\varepsilon)\right)$. In addition, the following holds.

i) If $\lambda+c \mathrm{e}^{-d_{1} T}-(2 d+\gamma) \leq 0$ then, for any $\varepsilon \in(0, \lambda-(d+\gamma))$ such that

$$
0<\varepsilon\left(1-\frac{d+\varepsilon+\gamma}{\lambda}\right)<-c e^{-d_{1} T}+d,
$$

holds, the endemic equilibrium $E_{\varepsilon}^{*}=\left(I^{*}(\varepsilon), N^{*}(\varepsilon)\right)$ is globally asymptotically stable in $X_{0}$. 
ii) If $\lambda+c \mathrm{e}^{-d_{1} T}-(2 d+\gamma)>0$ and $F_{1}\left(\varepsilon_{M}\right) \leq 1$, where

$$
F_{1}(\varepsilon)=\left(1-\frac{d+\varepsilon+\gamma}{\lambda}\right) \frac{(d+\varepsilon) e^{d_{1} T}-c}{d e^{d_{1} T}-c}, \text { for } \varepsilon \in(0, \lambda-(d+\gamma))
$$

and

$$
\varepsilon_{M}=\frac{1}{2}\left(\lambda+c e^{-d_{1} T}-(2 d+\gamma)\right)
$$

then, for any $\varepsilon \in(0, \lambda-(d+\gamma))$ such that (4.3) holds, the endemic equilibrium $E_{\varepsilon}^{*}=\left(I^{*}(\varepsilon), N^{*}(\varepsilon)\right)$ is globally asymptotically stable in $X_{0}$.

iii) If $\lambda+c \mathrm{e}^{-d_{1} T}-(2 d+\gamma)>0$ and $F_{1}\left(\varepsilon_{M}\right)>1$, then, there exist two positive solutions, $0<\varepsilon_{1}<\varepsilon_{2}<\lambda-(d+\gamma)$, of $F_{1}(\varepsilon)-1=0$, and, for any $\varepsilon \in\left(0, \varepsilon_{1}\right] \cup\left[\varepsilon_{2}, \lambda-(d+\gamma)\right)$ such that (4.3) holds, the endemic equilibrium $E_{\varepsilon}^{*}=\left(I^{*}(\varepsilon), N^{*}(\varepsilon)\right)$ is globally asymptotically stable in $X_{0}$.

PROOF. At first, we see $G^{\prime}(N) \mathrm{e}^{-d_{1} T}=c \mathrm{e}^{-d_{1} T}<d$ by (4.2). Hence, (3.2) in Theorem 3.5 holds.

Now we determine $\Omega_{1}$ defined by (3.19) in Theorem 3.5 for the cases i)- iii).

i) To determine $\Omega_{1}$, we consider the function $F_{1}(\varepsilon)$ defined by (4.4). It holds that $\lim _{\varepsilon \rightarrow+0} F_{1}(\varepsilon)=$ $1-\frac{1}{R_{0}(0)}<1$ if $R_{0}(0)>R_{0}(\varepsilon)>1$ and $\lim _{\varepsilon \rightarrow \lambda-(d+\gamma)} F_{1}(\varepsilon)=0$. Moreover, direct calculation gives

$$
\begin{aligned}
F_{1}^{\prime}(\varepsilon) & =-\frac{1}{\lambda} \frac{(d+\varepsilon) \mathrm{e}^{d_{1} T}-c}{d \mathrm{e}^{d_{1} T}-c}+\left(1-\frac{d+\varepsilon+\gamma}{\lambda}\right) \frac{\mathrm{e}^{d_{1} T}}{d \mathrm{e}^{d_{1} T}-c} \\
& =\frac{1}{\lambda\left(d \mathrm{e}^{d_{1} T}-c\right)}\left(\lambda+c \mathrm{e}^{-d_{1} T}-(2 d+2 \varepsilon+\gamma)\right) \mathrm{e}^{d_{1} T}
\end{aligned}
$$

and hence,

$$
\lim _{\varepsilon \rightarrow+0} F_{1}^{\prime}(\varepsilon)=\frac{1}{\lambda\left(d \mathrm{e}^{d_{1} T}-c\right)}\left(\lambda+c \mathrm{e}^{-d_{1} T}-(2 d+\gamma)\right) \mathrm{e}^{d_{1} T},
$$

follows. If $\lambda+c \mathrm{e}^{-d_{1} T}-(2 d+\gamma) \leq 0$ then we see that $F_{1}(\varepsilon)$ is nonincreasing from (4.5). Hence, $F_{1}(\varepsilon)<1$ for $\varepsilon \in(0, \lambda-(d+\gamma))$. Thus, $\Omega_{1}=(0, \lambda-(d+\gamma))$.

ii) From (4.5), if $\lambda+c \mathrm{e}^{-d_{1} T}-(2 d+\gamma)>0$, then $F_{1}(\varepsilon)$ attains a maximum at $\varepsilon_{M} \in(0, \lambda-$ $(d+\gamma))$. It is easy to see that if $F_{1}\left(\varepsilon_{M}\right) \leq 1$, then $F_{1}(\varepsilon)<1$ for $\varepsilon \in(0, \lambda-(d+\gamma))$. Thus, $\Omega_{1}=(0, \lambda-(d+\gamma))$.

iii) If $F_{1}\left(\varepsilon_{M}\right)>1$, then we see that $F_{1}(\varepsilon) \leq 1$ for any $\varepsilon \in\left(0, \varepsilon_{1}\right] \cup\left[\varepsilon_{2}, \lambda-(d+\gamma)\right)$. Thus, $\Omega_{1}=\left(0, \varepsilon_{1}\right] \cup\left[\varepsilon_{2}, \lambda-(d+\gamma)\right)$.

Finally, it follows

$$
-\max _{N \in\left[\underline{N}_{\varepsilon}, \bar{N}_{\varepsilon}\right]} G^{\prime}(N) \mathrm{e}^{-d_{1} T}+d=-c \mathrm{e}^{-d_{1} T}+d>0,
$$

and hence, by Theorem 3.5, we obtain the conclusion. The proof is complete.

We can determine $\varepsilon$ which guarantees the global asymptotic stability of the endemic equilibrium $E_{\varepsilon}^{*}=\left(I^{*}(\varepsilon), N^{*}(\varepsilon)\right)$ by (4.3). We also see that if $\varepsilon$ is sufficiently small or $R_{0}(\varepsilon)>1$ is sufficiently close to 1 (that is, $\varepsilon<\lambda-(d+\gamma)$ is sufficiently close to $\lambda-(d+\gamma)$ ) then (4.3) holds. From (4.3), we also determine $\bar{\varepsilon}$ in Theorem A 2. 
4.2. Case: $B_{1}(N)=b e^{-a N}$

In this subsection, we consider the SIS epidemic model with $B(N)=b \mathrm{e}^{-a N}$, that is,

$$
\left\{\begin{array}{l}
I^{\prime}(t)=\lambda(N(t)-I(t)) \frac{I(t)}{N(t)}-(d+\varepsilon+\gamma) I(t), \\
N^{\prime}(t)=b \mathrm{e}^{-a N(t-T)} N(t-T) \mathrm{e}^{-d_{1} T}-d N(t)-\varepsilon I(t) .
\end{array}\right.
$$

For (H1), we assume

$$
\lim _{N \rightarrow+0} B(N)=b>(d+\varepsilon) \mathrm{e}^{d_{1} T}>d \mathrm{e}^{d_{1} T}>\lim _{N \rightarrow+\infty} B(N)=0 .
$$

Then, (4.6) has the disease free equilibrium $E_{0}=\left(0, B^{-1}\left(d \mathrm{e}^{d_{1} T}\right)\right)$ and the endemic equilibrium $E_{\varepsilon}^{*}=\left(I^{*}(\varepsilon), N^{*}(\varepsilon)\right)$ if $R_{0}(\varepsilon)=\frac{\lambda}{d+\varepsilon+\gamma}>1$.

From Kuang [3, Corollary 4.3], the positive equilibrium $N^{*}(0)=\bar{N}_{\varepsilon}$ of

$$
N^{\prime}(t)=b \mathrm{e}^{-a N(t-T)} N(t-T) \mathrm{e}^{-d_{1} T}-d N(t),
$$

is globally asymptotically stable if

$$
\ln \frac{b}{d \mathrm{e}^{d_{1} T}}<2
$$

Hence, we assume (4.8) to ensure that $(\mathrm{H} 2)$ holds. Other conditions for the global stability were investigated by Cooke et al. [1].

On the other hand, by Theorem 2.1 , if $R_{0}(\varepsilon)>1$, then it holds

$$
0<\underline{N_{\varepsilon}} \leq \liminf _{t \rightarrow+\infty} N(t) \leq \limsup _{t \rightarrow+\infty} N(t) \leq \bar{N}_{\varepsilon}<+\infty,
$$

where

$$
\underline{N}_{\varepsilon}=\frac{1}{a} \ln \frac{b}{(d+\varepsilon) \mathrm{e}^{d_{1} T}}, \bar{N}_{\varepsilon}=\frac{1}{a} \ln \frac{b}{d \mathrm{e}^{d_{1} T}} .
$$

To present global stability results for (4.6), we introduce the following lemma.

Lemma 4.2 Let $G(N)=B_{1}(N) N, N \in(0,+\infty)$ where $B_{1}(N)=b e^{-a N}, b>0, a>0$, it holds that

$$
\begin{gathered}
G^{\prime}(N)=B_{1}(N)+B^{\prime}(N) N=(1-a N) B_{1}(N), \\
G^{\prime \prime}(N)=2 B_{1}^{\prime}(N)+B_{1}^{\prime \prime}(N) N=(a N-2) a B_{1}(N) .
\end{gathered}
$$

In particular, $G(N)$ is monotone increasing on $N \in\left(0, \frac{1}{a}\right]$ and decreasing on $N \in\left[\frac{1}{a},+\infty\right)$.

PROOF. We see $B_{1}^{\prime}(N)=-a B_{1}(N)$ and $B_{1}^{\prime \prime}(N)=a^{2} B_{1}(N)$. By direct calculation, we obtain the conclusion.

We see that if $\bar{N}_{\varepsilon} \leq \frac{1}{a}$, then $G^{\prime}(N) \geq 0$ for $N \in\left[\underline{N}_{\varepsilon}, \bar{N}_{\varepsilon}\right]$. In this case, we establish the following result from Theorem 3.5.

Theorem 4.3 Assume that (4.7) holds. If $R_{0}(\varepsilon)>1$, then (4.6) admits an endemic equilibrium $E_{\varepsilon}^{*}=\left(I^{*}(\varepsilon), N^{*}(\varepsilon)\right)$. Let $\hat{\varepsilon}_{1}$ be a unique positive solution of $g(\varepsilon)=0$, where

$$
g(\varepsilon)=(d+\varepsilon) \ln \frac{b}{(d+\varepsilon) e^{d_{1} T}}-\varepsilon \text { for } \varepsilon>0,
$$


and $\varepsilon_{M} \in(0, \lambda-(d+\gamma))$ be a unique positive solution of $f(\varepsilon)-1=0$, where

$$
f(\varepsilon)=-\ln \frac{b}{(d+\varepsilon) e^{d_{1} T}}+\frac{\lambda-(d+\varepsilon+\gamma)}{d+\varepsilon},
$$

if $\lim _{\mathcal{\varepsilon} \rightarrow+0} f(\varepsilon)=-\ln \frac{b}{d \mathrm{e}^{d_{1} T}}+\frac{\lambda-(d+\gamma)}{d}>0$. In addition, suppose that

$$
\ln \frac{b}{d \mathrm{e}^{d_{1} T}} \leq 1
$$

and $\varepsilon<\hat{\varepsilon}_{1}$, then the following holds.

i) If $-\ln \frac{b}{d^{d_{1} T}}+\frac{\lambda-(d+\gamma)}{d} \leq 0$, then for any $\varepsilon \in(0, \lambda-(d+\gamma)) \cap\left(0, \hat{\varepsilon}_{1}\right)$ such that

$$
0<\varepsilon\left(1-\frac{d+\varepsilon+\gamma}{\lambda}\right)<(d+\varepsilon) \ln \frac{b}{(d+\varepsilon) e^{d_{1} T}}-\varepsilon,
$$

the endemic equilibrium $E_{\varepsilon}^{*}=\left(I^{*}(\varepsilon), N^{*}(\varepsilon)\right)$ is globally asymptotically stable in $X_{0}$.

ii) If $-\ln \frac{b}{d^{d^{d_{1} T}}}+\frac{\lambda-(d+\gamma)}{d}>0$ and $F_{1}\left(\varepsilon_{M}\right) \leq 1$, where

$$
F_{1}(\varepsilon)=\left(1-\frac{d+\varepsilon+\gamma}{\lambda}\right) \frac{\ln \frac{b}{d e^{d_{1} T}}}{\ln \frac{b}{(d+\varepsilon) e^{d_{1} T}}} \text { for } \varepsilon \in(0, \lambda-(d+\gamma)),
$$

then, for any $\varepsilon \in(0, \lambda-(d+\gamma)) \cap\left(0, \hat{\varepsilon}_{1}\right)$ such that (4.14) holds, the endemic equilibrium $E_{\varepsilon}^{*}=$ $\left(I^{*}(\varepsilon), N^{*}(\varepsilon)\right)$ is globally asymptotically stable in $X_{0}$.

iii) If $-\ln \frac{b}{d \mathrm{e}^{d_{1} T}}+\frac{\lambda-(d+\gamma)}{d}>0$ and $F_{1}\left(\varepsilon_{M}\right)>1$, then there there exist two positive solutions $0<\varepsilon_{3}<\varepsilon_{4}<\lambda-(d+\gamma)$ of $F_{1}(\varepsilon)-1=0$, and, for any $\varepsilon \in\left(\left(0, \varepsilon_{3}\right] \cup\left[\varepsilon_{4}, \lambda-(d+\gamma)\right)\right) \cap\left(0, \hat{\varepsilon}_{1}\right)$ such that (4.14) holds, the endemic equilibrium $E_{\varepsilon}^{*}=\left(I^{*}(\varepsilon), N^{*}(\varepsilon)\right)$ is globally asymptotically stable in $X_{0}$.

PROOF. Assume that (4.13) holds and $\varepsilon<\hat{\varepsilon}_{1}$. We show $-\max _{N \in\left[\underline{N}_{\varepsilon}, \bar{N}_{\varepsilon}\right]} G^{\prime}(N)+d>0$ to verify (3.2) in Theorem 3.5. From (4.11) in Lemma 4.2, $G^{\prime}(N)$ is monotone decreasing on $N \in\left[\underline{N}_{\varepsilon}, \bar{N}_{\varepsilon}\right]$. Then, it holds that

$$
\begin{aligned}
-\max _{N \in\left[\underline{N}_{\varepsilon}, \bar{N}_{\varepsilon}\right]}\left\{G^{\prime}(N) \mathrm{e}^{-d_{1} T}\right\}+d & =-G^{\prime}\left(\underline{N}_{\varepsilon}\right) \mathrm{e}^{-d_{1} T}+d \\
& =-\left(1-a \underline{N}_{\varepsilon}\right) B\left(\underline{N}_{\varepsilon}\right) \mathrm{e}^{-d_{1} T}+d \\
& =-\left(1-\ln \frac{b}{(d+\varepsilon) \mathrm{e}^{d_{1} T}}\right)(d+\varepsilon)+d \\
& =(d+\varepsilon) \ln \frac{b}{(d+\varepsilon) \mathrm{e}^{d_{1} T}}-\varepsilon .
\end{aligned}
$$

Consider $g(\varepsilon)$ defined by (4.12). Since we have $\lim _{\varepsilon \rightarrow+0} g(\varepsilon)>0$ and, from (4.13), it follows

$$
g^{\prime}(\varepsilon)=\ln \frac{b}{(d+\varepsilon) \mathrm{e}^{d_{1} T}}-2<0,
$$

there exists $\hat{\varepsilon}_{1}$ such that $g\left(\hat{\varepsilon}_{1}\right)=0$ and $g(\varepsilon)>0$ for $\varepsilon<\hat{\varepsilon}_{1}$. Hence, $-\max _{N \in\left[\underline{N}_{\varepsilon}, \bar{N}_{\varepsilon}\right]} G^{\prime}(N)+d>0$ for $\varepsilon<\hat{\varepsilon}_{1}$ and (3.2) in Theorem 3.5 holds.

Now we determine $\Omega_{1}$ defined by (3.19) in Theorem 3.5 for i), ii) and iii). 
i) We claim $\Omega_{1}$ is $(0, \lambda-(d+\gamma))$. To determine $\Omega_{1}$, we consider $F_{1}(\varepsilon)$ defined by (4.15). It holds that $\lim _{\varepsilon \rightarrow+0} F_{1}(\varepsilon)=1-\frac{1}{R_{0}(0)}<1$ if $R_{0}(0)>R_{0}(\varepsilon)>1$ and $\lim _{\mathcal{\varepsilon} \rightarrow \lambda-(d+\gamma)} F_{1}(\varepsilon)=0$. Moreover, direct calculation gives

$$
\begin{aligned}
F_{1}^{\prime}(\varepsilon) & =\frac{\ln \frac{b}{d \mathrm{e}^{d_{1} T}}}{\left(\ln \frac{b}{(d+\varepsilon) \mathrm{e}^{d_{1} T}}\right)^{2}}\left[-\frac{1}{\lambda} \ln \frac{b}{(d+\varepsilon) \mathrm{e}^{d_{1} T}}+\left(1-\frac{d+\varepsilon+\gamma}{\lambda}\right)\left(\frac{1}{d+\varepsilon}\right)\right] \\
& =\frac{\ln \frac{b}{d_{\mathrm{e}^{d_{1} T}}}}{\lambda\left(\ln \frac{b}{(d+\varepsilon) \mathrm{e}^{d_{1} T}}\right)^{2}} f(\varepsilon) .
\end{aligned}
$$

We see

$$
f^{\prime}(\varepsilon)=\frac{1}{d+\varepsilon}+\frac{-(d+\varepsilon)-(\lambda-(d+\varepsilon+\gamma))}{(d+\varepsilon)^{2}}=\frac{-(\lambda-(d+\varepsilon+\gamma))}{(d+\varepsilon)^{2}}<0,
$$

if $R_{0}(\varepsilon)=\frac{\lambda}{d+\varepsilon+\gamma}>1$. Since we have

$$
\lim _{\varepsilon \rightarrow+0} F_{1}^{\prime}(\varepsilon)=\frac{\ln \frac{b}{d_{\mathrm{e}^{d_{1} T}}}}{\lambda\left(\ln \frac{b}{(d+\varepsilon) \mathrm{e}^{d_{1} T}}\right)^{2}}\left(-\ln \frac{b}{d \mathrm{e}^{d_{1} T}}+\frac{\lambda-(d+\gamma)}{d}\right) \leq 0,
$$

there does not exist $\hat{\varepsilon}>0$ such that $F_{1}^{\prime}(\hat{\varepsilon})=0$ and $F_{1}(\varepsilon)$ is nonincreasing. Hence, $F_{1}(\varepsilon)<1$ for $\varepsilon \in(0, \lambda-(d+\gamma))$. Thus, $\Omega_{1}=(0, \lambda-(d+\gamma))$.

ii) Since we have $\lim _{\varepsilon \rightarrow+0} f(\varepsilon)>0$ and $\lim _{\varepsilon \rightarrow \lambda-(d+\gamma)} f(\varepsilon)<0$, there exists $\varepsilon_{M}$ such that $F_{1}\left(\varepsilon_{M}\right)=\max _{0<\varepsilon<\lambda-(d+\gamma)} F_{1}(\varepsilon)$. Therefore, if $F_{1}\left(\varepsilon_{M}\right) \leq 1$, then $F_{1}(\varepsilon)<1$ for $\varepsilon \in(0, \lambda-(d+$ $\gamma)$ ). Thus, $\Omega_{1}=(0, \lambda-(d+\gamma))$.

iii) If $F_{1}\left(\varepsilon_{M}\right)>1$, then we see that $F_{1}(\varepsilon) \leq 1$ for any $\varepsilon \in\left(0, \varepsilon_{3}\right] \cup\left[\varepsilon_{4}, \lambda-(d+\gamma)\right)$. Thus, $\Omega_{1}=\left(0, \varepsilon_{3}\right] \cup\left[\varepsilon_{4}, \lambda-(d+\gamma)\right)$.

By Theorem 3.5, we obtain the conclusion and the proof is complete.

If $\underline{N}_{\varepsilon} \geq \frac{1}{a}$, then $G^{\prime}(N) \leq 0$ for $N \in\left[\underline{N}_{\varepsilon}, \bar{N}_{\varepsilon}\right]$. In this case, we establish the following result from Theorem 3.8.

Theorem 4.4 Assume that (4.7) and $R_{0}(\varepsilon)>1$ then (4.6) admits an endemic equilibrium $E_{\varepsilon}^{*}=$ $\left(I^{*}(\varepsilon), N^{*}(\varepsilon)\right)$. In addition, suppose that

$$
\begin{gathered}
1<\ln \frac{b}{d \mathrm{e}^{d_{1} T}}<2, \\
\varepsilon \leq \hat{\varepsilon}_{2}=b e^{-1-d_{1} T}-d,
\end{gathered}
$$

and

$$
\ln \frac{b}{d e^{d_{1} T}} \leq \min \left\{\frac{1}{1-\frac{d+\gamma}{\lambda}}, \frac{\lambda}{d+\gamma}\right\} .
$$

Then there exists $\Omega_{2}=\left\{\varepsilon \in(0, \lambda-(d+\gamma)) \mid F_{j}(\varepsilon) \leq 1, j=2,3\right\}$ where

$$
F_{2}(\varepsilon)=\frac{d \frac{1}{a} \ln \frac{b}{(d+\varepsilon) e^{d_{1} T}}}{(d-\tilde{\varepsilon}) \frac{1}{a} \ln \frac{b}{d e^{d_{1} T}}}, F_{3}(\varepsilon)=\frac{(d+\varepsilon-\tilde{\varepsilon}) \frac{1}{a} \ln \frac{b}{(d+\varepsilon) e^{d_{1} T}}}{d \frac{1}{a} \ln \frac{b}{d e^{d_{1} T}}} \text { for } \varepsilon \in(0, \lambda-(d+\gamma)),
$$


with $\tilde{\varepsilon}<d$ and, for any $\varepsilon \in \Omega_{2} \cap\left(0, \hat{\varepsilon}_{2}\right]$ such that

$$
0<\varepsilon\left(1-\frac{d+\varepsilon+\gamma}{\lambda}\right)<d\left(2-\ln \frac{b}{d e^{d_{1} T}}\right) \text { for } \ln \frac{b}{d e^{d_{1} T}}<2,
$$

the endemic equilibrium $E_{\varepsilon}^{*}=\left(I^{*}(\varepsilon), N^{*}(\varepsilon)\right)$ is globally asymptotically stable in $X_{0}$.

PROOF. From (4.17), we see $b>d \mathrm{e}^{d_{1} T+1}$ from which $b \mathrm{e}^{-1-d_{1} T}-d>0$ follows. Assume that $\varepsilon \leq \hat{\varepsilon}_{2}=b \mathrm{e}^{-1-d_{1} T}-d$. Then, from (4.17), it holds

$$
1 \leq \ln \frac{b}{(d+\varepsilon) \mathrm{e}^{d_{1} T}}<\ln \frac{b}{d \mathrm{e}^{d_{1} T}}<2 .
$$

This gives $\underline{N}_{\varepsilon} \geq \frac{1}{a}$ and hence, we see $G^{\prime}(N) \leq 0$ for $N \in\left[\underline{N}_{\varepsilon}, \bar{N}_{\varepsilon}\right]$ from (4.10) in Lemma 4.2. Thus, (3.25) in Theorem 3.8 holds.

Next we show the existence of $\Omega_{2}$ in Theorem 3.8. We see $\lim _{\varepsilon \rightarrow 0} F_{j}(\varepsilon)=1, j=2,3$. Now we compute $F_{j}^{\prime}(\varepsilon)$. By direct calculation, we obtain

$$
F_{2}^{\prime}(\varepsilon)=\frac{d}{\ln \frac{b}{d \mathrm{e}^{d_{1} T}}}\left[\frac{\left(-\frac{1}{d+\varepsilon}\right)(d-\tilde{\varepsilon})-\ln \frac{b}{(d+\varepsilon) \mathrm{e}^{d_{1} T}}\left(\frac{d+2 \varepsilon+\gamma}{\lambda}-1\right)}{(d-\tilde{\varepsilon})^{2}}\right],
$$

and

$$
\lim _{\varepsilon \rightarrow+0} F_{2}^{\prime}(\varepsilon)=\frac{1}{d \ln \frac{b}{d \mathrm{e}^{d_{1} T}}}\left[\ln \frac{b}{d \mathrm{e}^{d_{1} T}}\left(1-\frac{d+\gamma}{\lambda}\right)-1\right] .
$$

Similarly, we obtain

$$
F_{3}^{\prime}(\varepsilon)=\frac{1}{d \ln \frac{b}{d \mathrm{e}^{d_{1} T}}}\left[\left(\frac{d+2 \varepsilon+\gamma}{\lambda}\right) \ln \frac{b}{(d+\varepsilon) \mathrm{e}^{d_{1} T}}-(d+\varepsilon-\tilde{\varepsilon}) \frac{1}{d+\varepsilon}\right],
$$

and

$$
\lim _{\varepsilon \rightarrow+0} F_{3}^{\prime}(\varepsilon)=\frac{1}{d \ln \frac{b}{d \mathrm{e}^{d_{1} T}}}\left[\frac{d+\gamma}{\lambda} \ln \frac{b}{d \mathrm{e}^{d_{1} T}}-1\right] .
$$

Therefore, (F1) in Theorem 3.8 holds from (4.19) and we see $\Omega_{2} \neq \emptyset$.

Next, we calculate $\min _{N \in\left[\underline{N}_{\varepsilon}, \bar{N}_{\varepsilon}\right]}\left\{G^{\prime}(N) \mathrm{e}^{-d_{1} T}\right\}+d$ in Theorem 3.8. We have that $G^{\prime}(N)$ is monotone decreasing on $N \in\left[\underline{N}_{\varepsilon}, \bar{N}_{\varepsilon}\right]$ by (4.11) in Lemma 4.2. Hence, it holds that

$$
\begin{aligned}
\min _{N \in\left[\underline{N}_{\varepsilon}, \bar{N}_{\varepsilon}\right]}\left\{G^{\prime}(N) \mathrm{e}^{-d_{1} T}\right\}+d & =\left(1-a \bar{N}_{\varepsilon}\right) b \mathrm{e}^{-a \bar{N}_{\varepsilon}} \mathrm{e}^{-d_{1} T}+d \\
& =\left(1-a \bar{N}_{\varepsilon}\right) d+d \\
& =d\left(2-a \bar{N}_{\varepsilon}\right) \\
& =d\left(2-\ln \frac{b}{d \mathrm{e}^{d_{1} T}}\right)>0,
\end{aligned}
$$

Finally, by Theorem 3.8, we obtain the conclusion.

Theorems 4.3 and 4.4 show that it is possible that the endemic equilibrium $E_{\varepsilon}^{*}=\left(I^{*}(\varepsilon), N^{*}(\varepsilon)\right)$ is globally asymptotically stable for the corresponding case of (4.8). In fact, we obtain the global stability results for $\bar{N}_{\varepsilon}<\frac{2}{a}$, that is, (4.8). From some numerical simulations, every solution seems 
to converge to the endemic equilibrium $E_{\varepsilon}^{*}$ if $R_{0}(\varepsilon)>1$ and (4.8) holds. If (4.8) does not holds, periodic solution may arise (see also Cooke et al. [1]). Bifurcation analysis was carried out by Wei and Zou [4].

Finally, as a numerical example for Theorem 4.4, we consider (4.6) with $b=1.5, a=0.3, \lambda=$ $1.4, d=0.3, \gamma=0.3$ and $d_{1} T=0$. We vary the parameter $\varepsilon$. We see

$$
R_{0}(\varepsilon)=\frac{\lambda}{d+\varepsilon+\gamma}=\frac{1.4}{0.3+\varepsilon+0.3}>1, \text { if } \varepsilon<0.8
$$

from (1.5). If $\varepsilon<0.8$, then (4.7) holds, since

$$
b=1.5>d+\varepsilon=0.3+\varepsilon \text { if } \varepsilon<0.8,
$$

follows. Hence, there exists an endemic equilibrium $E_{\varepsilon}^{*}=\left(I^{*}(\varepsilon), N^{*}(\varepsilon)\right)$ for (4.6).

Since we have

$$
1<\ln \frac{b}{d}=\ln \frac{1.5}{0.3} \approx 1.6094 \cdots<2 .
$$

(4.17) holds. For (4.18), we see

$$
\hat{\varepsilon}_{2}=b \mathrm{e}^{-1}-d=1.5 \mathrm{e}^{-1}-0.3=0.2518 \ldots
$$

Hence, we restrict $\varepsilon \leq 0.2518$. Further, it holds that

$$
\ln \frac{b}{d} \approx 1.6094 \leq \min \left\{\frac{1}{1-\frac{d+\gamma}{\lambda}}, \frac{\lambda}{d+\gamma}\right\}=\frac{1.4}{0.8}=1.75
$$

which implies (4.19) holds.

Consider the following functions $F_{j}(\varepsilon), j=2,3$.

$$
F_{2}(\varepsilon)=\frac{0.3 \ln \frac{1.5}{0.3+\varepsilon}}{\left(0.3-\varepsilon\left(1-\frac{0.3+\varepsilon+0.3}{1.4}\right)\right) \ln \frac{1.5}{0.3}}, F_{3}(\varepsilon)=\frac{\left(0.3+\varepsilon-\varepsilon\left(1-\frac{0.3+\varepsilon+0.3}{1.4}\right)\right) \ln \frac{1.5}{0.3+\varepsilon}}{0.3 \ln \frac{1.5}{0.3}} .
$$

We present the graph for $F_{j}(\varepsilon), j=2,3$ in Figure 3 . We see $F_{j}(\varepsilon) \leq 1, j=2,3$ for $\varepsilon \in(0, \lambda-$ $(d+\gamma))$ and hence, $\Omega_{2}=(0, \lambda-(d+\gamma))$.

Finally it follows that

$$
\max _{\varepsilon \in(0,0.2518]} \tilde{\varepsilon}=0.2518\left(1-\frac{0.3+0.2518+0.3}{1.4}\right) \approx 0.0985<d\left(2-\ln \frac{b}{d}\right) \approx 0.1171 .
$$

Consequently, by Theorem 4.4 , if $\varepsilon \leq 0.2518$, then the endemic equilibrium $E_{\varepsilon}^{*}$ is globally asymptotically stable.

\section{Discussion}

In this paper, we study the global asymptotic stability for a SIS epidemic model with maturation delay proposed by Cooke et al. [1]. Cooke et al. [1] showed that $R_{0}(\varepsilon)$ works as a global threshold parameter if there is no maturation delay $(T=0)$ or if the disease does not induce the death of the infective $(\varepsilon=0)$ under the assumptions (H1) and (H2). More precisely, if $R_{0}(\varepsilon)<1$ then the disease free equilibrium $E_{0}$ is globally asymptotically stable and if $R_{0}(\varepsilon)>1$ then the endemic equilibrium $E_{\varepsilon}^{*}$ exists and is globally asymptotically stable in these cases.

Zhao and Zou [5] also studied the global dynamics of (1.3). They established that the basic reproduction number $R_{0}(\varepsilon)$ works as a threshold parameter which determines the extinction of the 


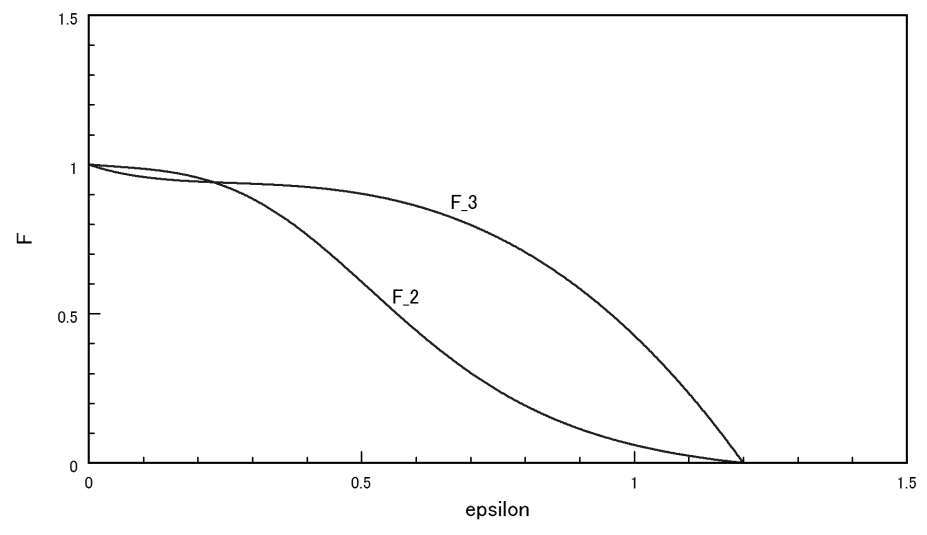

Fig. 3. Graph trajectory of $F_{j}(\varepsilon), j=2,3$

disease, even if the disease causes the death of the population $(\varepsilon>0)$. Moreover, they studied the global attractivity of the endemic equilibrium $E_{\varepsilon}^{*}$ by using a perturbation theory and showed that if $\varepsilon$ is small enough, then the endemic equilibrium $E_{\varepsilon}^{*}$ is still globally attractive (see Theorems A 1 and A 2).

However, the dynamics of (1.3) is not completely understood, in particular, for $R_{0}(\varepsilon)>1$. To obtain a detailed information about the dynamics when the disease is endemic, we investigate the global stability of the endemic equilibrium $E_{\varepsilon}^{*}$ by monotone iterative method. Sufficient conditions for the global stability of the endemic equilibrium $E_{\varepsilon}^{*}$ of (1.3) are established in Theorems 3.5 and 3.8. From these results, we can determine $\bar{\varepsilon}$ in Theorem A 2 by Zhao and Zou [5]. Our results show that $G^{\prime}(N)=(B(N) N)^{\prime}$ is important to determine the global stability for (1.3). In Section 4, from Theorems 3.5 and 3.8, we establish the global stability of the endemic equilibrium $E_{\varepsilon}^{*}$ for the birth rate functions $B_{3}(N)=\frac{A}{N}+c$ and $B_{1}(N)=b \mathrm{e}^{-a N}$ (see Theorems 4.1, 4.3 and 4.4). For these cases, we obtain the disease induced death rate $\varepsilon$ which guarantees the global stability of the endemic equilibrium $E_{\varepsilon}^{*}$. We also introduce a numerical example. From these results, we determine $\bar{\varepsilon}$ in Theorem A 2 .

For $B_{3}(N)=\frac{A}{N}+c$, if the disease induced death rate $\varepsilon$ is sufficiently small or the basic reproduction number $R_{0}(\varepsilon)>1$ is close to 1 enough, then the condition in Theorem 4.1 holds. This implies that the endemic equilibrium $E_{\varepsilon}^{*}$ is also globally asymptotic stable even if the disease induced death rate $\varepsilon$ is sufficiently large. From this, one may conjecture that $\varepsilon$ does not influence the global stability of the endemic equilibrium $E_{\varepsilon}^{*}$, but this is still an open problem. For $B_{1}(N)=b \mathrm{e}^{-a N}$, it is possible that the endemic equilibrium $E_{\varepsilon}^{*}$ is globally asymptotic stable if (4.8) holds. Is the endemic equilibrium $E_{\varepsilon}^{*}$ always globally asymptotically stable if $R_{0}(\varepsilon)>1$ and (4.8) holds? This problem also needs further study.

\section{Acknowledgements}

The authors are grateful to the two referees for their constructive comments which led to a significant improvement of the original manuscript. 


\section{References}

[1] K. Cooke, P. van den Driessche, and X. Zou, Interaction of maturation delay and nonlinear birth in population and epidemic models, J. Math. Biol. 39 (1999), no. 4, 332-352. MR MR1727839 (2001b:92040)

[2] H. I. Freedman and K. Gopalsamy, Global stability in time-delayed single-species dynamics, Bull. Math. Biol. 48 (1986), no. 5-6, 485-492. MR MR884745 (88f:92031)

[3] Yang Kuang, Global attractivity and periodic solutions in delay-differential equations related to models in physiology and population biology, Japan J. Indust. Appl. Math. 9 (1992), no. 2, 205-238. MR MR1170722 (93j:34103)

[4] Junjie Wei and Xingfu Zou, Bifurcation analysis of a population model and the resulting SIS epidemic model with delay, J. Comput. Appl. Math. 197 (2006), no. 1, 169-187. MR MR2256059 (2007i:34114)

[5] Xiao-Qiang Zhao and Xingfu Zou, Threshold dynamics in a delayed SIS epidemic model, J. Math. Anal. Appl. 257 (2001), no. 2, 282-291. MR MR1827321 (2002c:92036) 